\title{
ANTAL KovÁts* \\ DAS BILD UNGARISCHER SCHÜLER VON DEN DEUTSCHSPRACHIGEN LÄNDERN UND VON DEN DEUTSCHEN
}

(Erhalten: 17. Februar 2010; angenommen: 14. Juni 2010)

\begin{abstract}
Die vorliegende Arbeit stellt die Ergebnisse der Fortsetzung einer vor 25 Jahren durchgeführten Untersuchung zum Thema Deutschbild der Schüler der 8. Grundschulklasse in Ungarn dar. Das Ziel der Untersuchung war festzustellen, was für ein Bild die Grundschulabsolventen von den deutschsprachigen Ländern und Menschen haben bzw. wie dieses Bild von ihren Informationsquellen wie Schule, Elternhaus, Medien und Bekannten beeinflusst wird. Die durchgeführte Erhebung bietet auch der Bildungspolitik Rückschlüsse darüber, wie die in den verschiedenen Fächern und anderen Informationsquellen vermittelten Kenntnisse herüberkommen bzw. wie sie von den Schülern synthetisiert in ihren Wissensstand aufgenommen werden. Das Thema ist aktuell geworden, da in diesem Vierteljahrhundert - nach der politischen Wende in den Jahren 1989-1990 - viele Veränderungen in den Informationsquellen, die das Bild über die Deutschen beeinflussen, eingetreten sind. Der Einfluss der Medien ist allgemein stärker geworden, das gesellschaftliche Umfeld hat sich geändert, und auch im schulischen Unterricht wurden neue Schwerpunkte gesetzt. Es ging bei dieser Untersuchung teilweise um eine Bestandsaufnahme davon, wie das Bild der Achtklässler über die Deutschen und die deutschsprachigen Ländern heute ist, andererseits aber auch um einen Vergleich gegenüber den vor 25 Jahren gewonnenen Ergebnissen. In diesem Beitrag werden nach der Problemstellung und der Darstellung der Vorbereitungen für die Erhebung die Überlegungen bei der Auswahl der Befragten, die Fragen und ihre Anliegen, die Prinzipien bei der Auswertung der ausgefüllten Fragebögen sowie die statistischen Ergebnisse erörtert. Nach einem Vergleich der Ergebnisse von 1980 mit denen im Jahre 2005 erfolgt eine Gegenüberstellung von erfassten Schülermeinungen, die auf Stereotypen hindeuten, und anderen, in Ungarn und in weiteren Ländern gewonnenen einschlägigen Erkenntnissen. Zum Schluss werden die in den Schulen verwendeten relevanten Lehrbücher im Spiegel der gestellten Fragen betrachtet, um zu sehen, wie weit diese zu Kenntnissen und Unkenntnissen in den untersuchten Fragen beitragen konnten. Die Fragen der zu den beiden Erhebungen

\footnotetext{
* Antal Kováts, Institut für Mentalhygiene, Semmelweis-Universität, Nagyvárad tér 4., 19. em., H-1089 Budapest, Ungarn; talford@t-online.hu.
} 
benutzten Fragebögen wurden weitgehend gleichgehalten, um einen Vergleich zu ermöglichen. Die Fragen bezogen sich zum Teil nicht nur auf schulische Kenntnisse in Geographie, Geschichte und auf Themen der allgemeinen Bildung, sondern auch auf Emotionen der Schüler.

Schlüsselbegriffe: Kenntnisse, Stereotypen, Unterrichtseffektivität, Emotionen, synthetisierendes Lernen, Schüler, Deutsche, deutschsprachige Länder, Erhebung

The View Hungarian School Children Have of Germans and German-Speaking Countries: The paper presents the results of a follow-up study to a survey carried out 25 years earlier, which looked at the view of Germans held by Hungarian $8^{\text {th }}$ grade primary school children. In addition to determining how children at the end of their primary school studies viewed German-speaking countries and people, the purpose of the study was also to examine the degree to which their sources of information e.g., school, family, media and people they know influenced this picture. The assessment that was carried out also provided feedback to education policymakers regarding the efficiency of the transfer of knowledge to children via various subjects and other information sources, and about how this knowledge is incorporated into their larger set of knowledge. Besides the political changes of 1989-1990, the time is right to revisit the topic because of the amount of changes in the sources of information that influence the views held of Germans over this quarter of a century. In general, the influence of the media has become stronger, the social environment has changed, and there are also new points of emphasis in teaching. In part, the study served as an assessment of the current situation concerning the view school children have of Germans and German-speaking countries, while on the other hand, the aim was to compare these with the results from 25 years ago. After introducing the problem and the preparations for conducting the survey, the paper presents the considerations for selecting respondents, the questions and their purpose, the principles employed in evaluating the completed questionnaires, and the statistical results. Following a comparison of the results from 1980 and 2005, the stereotypical opinion of school children is compared to other similar results from Hungary and abroad. In closing, a review of relevant textbooks used by schools is presented in light of the questions posed. This was done in order to determine to what extent these might have contributed to the knowledge or lack thereof identified in relation to the questions examined. Differences between the questions used in the two questionnaires were minimised, in order to ensure that the responses could be compared. Some of the questions were not related to knowledge gained within the curriculum, in the fields of geography, history, and general knowledge, but rather focused on the emotions of school children regarding the issue.

Keywords: knowledge, stereotypes, efficiency of teaching, emotions, synthesizing learning, school children, Germans, German-speaking countries, survey

\section{Einleitung}

1979-1980 wurde eine Erhebung zur Untersuchung des Bildes durchgeführt, das die Schüler der 8. Grundschulklasse in Ungarn von den Deutschen und den deutschsprachigen Ländern haben. Als Germanist interessierte ich mich damals dafür, was die Schüler über das Deutschtum wissen, was für Gefühle sie gegenüber Deutschen haben, was die Determinanten ihres Deutschbildes sind, wovon ihr Bild über die Deutschen am meisten beeinflusst wird. Ich habe diese Erhebung mit der gleichen Frage- 
stellung nach 25 Jahren wiederholt. In der Zwischenzeit gab es eine politische Wende in Ungarn, welche auch die in der Schule vermittelten Informationen über die Deutschen geändert hat. Ich hielt es für berechtigt, nun wieder die Frage zu stellen, ob sich die Kenntnisse und die Einstellung der Schüler zum Thema „Deutsche“, „deutschsprachige Länder" sowie die damit zusammenhängenden Stereotypen verändert haben. Nicht nur der Unterrichtsstoff hat sich geändert, sondern auch die gesellschaftliche Umgebung und die Kultur. Heutzutage werden zum Beispiel kaum Kriegsfilme über den 2. Weltkrieg gespielt. DDR-Touristen am Plattensee gibt es auch nicht mehr. Dafür aber kann jeder frei in die deutschsprachigen Länder reisen, und die Jugendlichen bekommen viel mehr und vor allem ungefilterte Informationen über das Ausland als vor einem Vierteljahrhundert.

In der ersten Erhebung 1979-80 wurden die Schüler nach Emotionen und Kenntnissen gefragt. Letztere waren durch den Unterricht in der Schule, das Elternhaus und durch andere Erlebnisse wie Kriegsfilme oder attraktive Autos aus Deutschland am meisten beeinflusst. Um einen Vergleich ziehen zu können, habe ich mich bemüht, die meisten Fragen und Aufgaben der damals verteilten Fragebögen beizubehalten. Nur einige Punkte, die wegen der Änderung des Unterrichtsstoffes nicht mehr aktuell sind, wurden gestrichen. Ebenso musste auch eine neue Landkarte Europas gezeichnet werden, da neue Landesgrenzen im früheren Jugoslawien und der ehemaligen Tschechoslowakei entstanden sind.

Da bis jetzt Untersuchungen mit gleicher Fragestellung und Komplexität meines Wissens nicht vorliegen, werden zu einzelnen Teilfragen zum Vergleich einschlägige Ergebnisse und Erkenntnisse aus der neueren Literatur herangezogen.

\section{Problemstellung}

Diese Arbeit beruht auf einer Erhebung bei Schülern der 8. Klassen der Grundschule. Dieser Schultyp hat die Aufgabe, eine allgemeine Ausbildung zu geben. Die Schüler der 8. Klasse verfügen also über die Kenntnisse, die die Gesellschaft von jedem Bürger erwartet, sie sind aber auch vom Elternhaus nicht unabhängig. Die vierzehnjährigen Jugendlichen sind sehr sensibel und stehen allen Eindrücken der Umwelt aufgeschlossen gegenüber. Dazu gehören auch Völker und Länder, die sich um Ungarn herum befinden, also auch die deutschsprachigen.

Folgendes sollten die gestellten Fragen herausfinden:

- Wie viel Prozent der Schüler wissen, welche die deutschsprachigen Länder in Europa sind?

- Welche von Deutschen bewohnten Gebiete kennen sie noch?

- Wissen unsere Schüler ungefähr, wie viele Einwohner deutscher Nationalität Ungarn hat? Kennen sie von Ungarndeutschen bewohnte Gebiete? Wenn ja, woher?

- Was bedeutet für sie das Wort „Deutsch“?

- Wodurch ist Ihr Deutschbild am meisten beeinflusst?

- Was halten sie von den Deutschen im Unterschied zu den eigenen Landsleuten? 
- Was wissen Schüler der 8. Klasse über berühmte Persönlichkeiten des deutschen Volkes, über die sie im Laufe der allgemeinen Grundbildung gehört und gelesen haben sollten? Sind ihre Kenntnisse von ihrer Schule abhängig, oder zeigen sich auch andere Einflüsse?

- Wie viel Prozent der befragten Schüler waren selbst schon in einem deutschsprachigen Land? Wo waren sie? In welches deutschsprachige Land würden sie auf Grund ihrer Gefühle und Kenntnisse zunächst wieder fahren, wenn sie die freie Wahl hätten? Warum?

- Wie viel Prozent der Befragten lernen jetzt Deutsch?

- Gibt es in Hinblick auf Kenntnisse und Gefühle Unterschiede im Deutschbild zwischen den Deutschlernenden und Nicht-Deutschlernenden?

- Wie groß ist in dieser Hinsicht der Einfluss der Familie, des Elternhauses?

Die erzielten Erkenntnisse sollten auch Hinweise darauf geben, inwieweit die einzelnen Unterrichtsfächer zum Komplexwissen über ein konkretes Thema in Bezug auf deutschsprachige Länder und Leute beitragen können, und inwieweit Kenntnisse zu diesem Thema aus den einzelnen Unterrichtsfächern synthetisierend aufgenommen werden.

\section{Die Vorbereitungen für die Erhebung}

\section{1. Überlegungen bei der Auswahl}

Ein Fragebogen mit zehn Fragegruppen wurde zusammengestellt und in 120 Exemplaren vervielfältigt. (Ein Originalexemplar ist im Anhang beigefügt.) Die Erhebungen fanden sowohl vor 25 Jahren als auch jetzt, im Frühjahr 2005 in drei Schulen statt. Die Erhebung im Jahre 1980 fand in Innenbezirken von Budapest statt, die neue wurde in zwei Budapester Schulen und in einer Kecskeméter Schule durchgeführt. Die meisten Schüler leben in einer sehr informationsreichen Umwelt. Es war zu erwarten, dass sie eine überdurchschnittliche Leistung erbringen werden. Die bei ihnen auftauchenden Mängel melden sich bei benachteiligten Kindern höchstwahrscheinlich in viel größerem Maße. Etwa die Hälfte der befragten Schüler lernt in der Schule Deutsch.

\subsection{Die Fragen und das Anliegen der einzelnen Fragen}

1. Frage: „Europas Landkarte ist auf dem beigefügten Blatt zu sehen. In welchen auf der Landkarte zu sehenden Ländern leben deines Wissens Leute, die Deutsch als Muttersprache sprechen?“

Die Namen der einzelnen Länder waren auf der Blindkarte nicht angegeben. Die Schüler sahen nur die Grenzlinien der Staaten. Sie sollten diese Aufgabe vor allem auf Grund ihrer Geographiekenntnisse lösen. Das Ziel der Aufgabe war zu erfahren, ob die Schüler der 8. Klasse fähig sind, das Deutschtum geographisch einzuordnen. 
2. Frage:

A) „Auf dem Beilageblatt 2 ist Ungarns Landkarte zu sehen. Kennst du solche Gebiete (Städte, Dörfer), wo Menschen deutscher Nationalität ('Schwaben') leben? Wenn ja, bezeichne die Gebiete durch Schattierung!"“

Diese Aufgabe appelliert an Geographiekenntnisse und an Kenntnisse aus anderen Informationsquellen. Das Ziel der Aufgabe: Wie bekannt sind die von Deutschen bewohnten Gebiete unter den Schülern?

B) „Wie viele Einwohner deutscher Nationalität hat Ungarn deiner Meinung nach? Unterstreiche die Zahl, die du für richtig hältst!“ $(1.000,15.000$, 220.000, 1.000.000)

Die Schüler erfuhren in der Geographiestunde, dass die nationalen Minderheiten etwa zwei Prozent der Gesamtbevölkerung ausmachen. Es sollte ihnen auch bekannt sein, dass die größte nationale Minderheit in Ungarn (ohne Romas) die deutsche ist. So konnte man erwarten, dass logisch Denkende auf 220.000 tippen, obwohl die Zahl nicht genau sein konnte, da sie auf früheren statistischen Daten beruht.

C) „Hast du schon von 'Schwaben' gehört? Wenn ja, wo? Unterstreiche die Antwort!“ (Eltern, Großeltern, Schule, Freunde, Fernsehen, Radio, Zeitung, anderswo und zwar:)

Das Ziel der Frage ist zu erfahren, wodurch die Kenntnisse und Meinungen der Schüler über die Ungarndeutschen (Schwaben) am meisten beeinflusst sind.

3. Frage: „Was fällt dir an der ersten, zweiten und dritten Stelle ein, wenn du das Wort 'deutsch' hörst? Nummeriere deine Antwort!" (der deutsche Fußball - Autos (Mercedes, BMW, VW) - deutsche Komponisten - deutsche Schriftsteller, Dichter - deine Freunde, Bekannten - der zweite Weltkrieg - anderes und zwar:)

Schon allein diese einzige Frage könnte man sehr vielfältig bearbeiten. Das Hauptziel der Frage war es zu erfahren, ob die Meinung der Schüler über das Deutschtum vor allem von den Konsumgütern, Sportergebnissen, vom kulturellen Erbe, von persönlichen Beziehungen oder durch Kriegsfilme und Kriegsromane am meisten beeinflusst ist.

4. Frage: „Wo hast du am meisten von Deutschen gehört; unterstreiche!“ (zu Hause, von den Eltern, Großeltern, Geschwistern - in der Schule - aus Büchern aus Filmen - anderswo, und zwar:)

Die Antworten sollten zeigen, wodurch die Meinung der Schüler über das Deutschtum am meisten beeinflusst ist. Während Frage 3. nach einer Wertreihenfolge fragt, möchte diese Frage eine Antwort darauf finden, aus welchen Informationsquellen die Schüler ihr Deutschbild gewinnen.

5. Frage:

A) „Was für Menschen sind deiner Meinung nach die Deutschen? Unterstreiche die Eigenschaften, die für die Deutschen charakteristisch sind! Wo du nicht wählen kannst, schreibe ein X!“ 


$\begin{array}{lll}\text { beweglich } & - & \text { schwerfällig } \\ \text { fleißig } & - & \text { faul } \\ \text { bescheiden } & - & \text { anmaßend } \\ \text { tapfer, mutig } & - & \text { feige, ängstlich } \\ \text { tolerant } & - & \text { intolerant } \\ \text { phantasievoll } & - & \text { phantasielos } \\ \text { großzügig } & - & \text { kleinlich } \\ \text { geistreich } & - & \text { humorlos } \\ \text { aufrichtig } & - & \text { falsch } \\ \text { friedlich } & - & \text { streitsüchtig } \\ \text { kultiviert } & - & \text { primitiv } \\ \text { warmherzig } & - & \text { kalt }\end{array}$

B) „Jetzt nimm einen roten Stift und unterstreiche die Eigenschaften, die deiner Meinung nach auf die ungarischen Durchschnittsmenschen zutreffen.“

Von besonders großem Interesse ist es, welche Eigenschaften unterschiedlich sind. Diese Frage sucht eine Antwort darauf, was die befragten Schüler von den Deutschen halten.

6. Frage: „Namen berühmter Menschen sind zu lesen. Von ihnen konntest du auch in der Schule hören. Wodurch sind sie berühmt geworden?" (Beethoven, Luther, Bach, Hitler, Goethe, Thomas Mann, Gebrüder Grimm)

Beethoven, Luther, Bach und Hitler sind im Lehrstoff des Geschichtsbzw. Musik- und Gesangsunterrichts enthalten. Die anderen Persönlichkeiten werden in der Schule nur oberflächlich oder gar nicht behandelt, auch sie sollten den Schülern bekannt sein. Hier bietet sich eine gute Möglichkeit, u. a. die Effektivität des Geschichtsunterrichts zu kontrollieren.

7. Frage: „Warst du schon auf deutschem Boden? Wenn ja, zähle auf, in welchen Ländern!“

Die Frage erzielt eine Information über eine wichtige Quelle der Meinung vorausgesetzt, dass die bisherigen Aussagen auf persönlichen Erfahrungen beruhen.

8. Frage: „Wenn du jetzt in ein deutschsprachiges Land fahren könntest, wo würdest du hinfahren? Warum?" Eine weitere Frage nach den Gefühlen der Schüler: „Mit welchem Anliegen würden die befragten Kinder eine Reise in ein deutschsprachiges Land unternehmen? Was treibt sie gerade in dieses Land?“

9. Frage: „Lernst du Deutsch?“ (Ja - nein)

Hier wird nach der aktuellen Situation gefragt.

10. Frage: „Kann jemand bei euch zu Hause Deutsch? Wenn ja, wer?“ (Großeltern, Eltern, Geschwister, sonstige)

Auch die Familie spielt eine bedeutende Rolle bei der Meinungsbildung der Schüler der 8. Klasse. Wer kann nun dem Wissen der Kinder zufolge in der Familie Deutsch?

Zum Schluss wird noch nach dem Beruf der Eltern gefragt, um zu erfahren, ob es einen Zusammenhang zwischen dem Beruf der Eltern und den Kenntnissen und Gefühlen der Kinder in Bezug auf dieses Thema gibt. Bei den Fragen 1., 2. und 6., die 
nach Kenntnissen fragen, bietet sich die Möglichkeit, die drei Schulen zu vergleichen, um zu erfahren, ob die Antworten bzw. Kenntnisse der Schüler von der Schule bzw. vom Unterricht abhängig sind. Die Erhebung wurde in ungarischer Sprache durchgeführt.

\section{3. Überlegungen bei der Auswertung der ausgefüllten Fragebögen}

- Bewertung der Frage 1. (Landkarte): Bei der Bewertung der Lösung dieser Aufgabe wurden drei Antworttypen unterschieden:

1. Die drei deutschsprachigen Länder wurden richtig eingezeichnet.

2. Außer den drei Ländern wurden noch weitere von Deutschen bewohnte Gebiete angegeben.

3. Falsche oder zu wenig Gebiete wurden angegeben.

Es wurde geprüft, wie viele Kinder insgesamt und pro Schule zu den einzelnen Antworttypen gehören. Um zu erfahren, ob der Deutschunterricht hier eine Rolle spielt, wurden die Leistungen der Deutsch lernenden Klassen auch gesondert untersucht.

- Bewertung der Frage 2. (Blindkarte) bzw. der zweiten Aufgabengruppe: Die Antworten wurden bei dem Punkt (A) in vier Gruppen eingeordnet:

1. Ein Gebiet wurde richtig eingezeichnet.

2. Zwei oder mehrere Gebiete wurden richtig eingezeichnet.

3. Neben den richtigen gab es auch falsche Schattierungen.

4. Falsche oder keine Antwort.

Hier wurden das Gesamtergebnis, das Ergebnis der einzelnen Schulen und gesondert das Ergebnis der Deutsch lernenden Klassen geprüft. Das gilt auch für den Punkt (B). Beim Punkt (C) wurde nur das Gesamtergebnis geprüft.

- Bewertung der Frage 3.: Die Antworten waren hier nur in ihrer Gesamtwertung interessant. Da dieser Punkt eine halbgeschlossene Frage war, könnten hier auch die bei ,anders, und zwar: “ angegebenen Antworten interessant sein.

- Analyse der Antworten auf die Frage 4.: Hier wurden die einzelnen Informationsquellen auch nach Häufigkeit in der Gesamtwertung geprüft (Tabelle 6).

- Analyse der Antworten auf die Frage 5.: Nur diejenige Eigenschaft wurde gezählt, wo sich ein Unterschied zeigte.

- Analyse und Bewertung der Antworten bei Frage 6.: Die ersten Namen (Beethoven, Luther, Bach und Hitler) fragen nach Grundkenntnissen, sie wurden also gesondert bewertet.

Für jede richtige Antwort wurde ein Punkt gegeben, so konnte man für die tadellose Lösung der ersten Hälfte der Aufgabe sechs Punkte bekommen. Für halbrichtige Antworten wurde je ein halber Punkt gegeben. Dieselbe Methode wurde auch bei den weiteren Namen (Goethe, Thomas Mann, Gebrüder Grimm) befolgt.

- Frage 7.: Es wurde insgesamt festgestellt, wie viele Schüler schon in den einzelnen Ländern waren. Die Proportion in den Deutsch lernenden Klassen wurde extra geprüft. 
- Frage 8.: Die Wunschreiseziele wurden global je nach Land registriert und die angegebenen Ursachen in ihrer Gesamtwertung aufgezählt.

- Frage 9.: Diese Frage war eine Alternativfrage. Es wurde gezählt, wie viel Prozent der Antwortenden in der Gesamtwertung Deutsch lernen.

- Frage 10.: Geprüft wurde hier, insgesamt wie viele Großeltern, Eltern und Geschwister der Kenntnis des Schülers zufolge Deutsch können. Es wurde auch zwischen Arbeiter- und Angestelltenfamilien und Angehörigen der Intelligenz unterschieden.

Zum Schluss wurde auch nach dem Beruf der Eltern gefragt. Zur Intelligenz wurden nur diejenigen gezählt, die angeblich einen geistig produktiven Beruf haben. Eine Fehlerquelle war hier die manchmal ungenaue Angabe des Berufes der Eltern.

Das Große Deutsche Wörterbuch bezeichnet die Intelligenz als Gesamtheit der geistig Schaffenden. Meiner Meinung nach ist aber die Zugehörigkeit zur Intelligenz nicht nur vom Beruf abhängig, sondern auch davon, ob man eine ,intellektuelle“ Lebensführung hat. Es handelt sich also um eine Lebensform. Im Rahmen dieser Erhebung war es aber unmöglich festzustellen, wie die Befragten zu Hause leben, wie viel ihrer kulturellen Kenntnisse die Schüler zu Hause im Elternhaus bekommen. Es wurde den Schülern vor der Verteilung der Fragebögen mitgeteilt, dass es um eine anonyme Erhebung gehe, über die eine wissenschaftliche Arbeit geschrieben werden soll. Die Schüler durften auch Fragen stellen.

\section{Die Ergebnisse der Erhebung}

\subsection{Die statistischen Ergebnisse}

Die Tabellen 1-3 zeigen die statistischen Ergebnisse der Fragen 1., 2. (A) und 2. (B). Insgesamt gesehen konnte weniger als die Hälfte der befragten Schüler drei deutschsprachige Länder richtig benennen und über die Hälfte (56\%) gab eine mangelhafte oder falsche Antwort an. Generell gilt, dass die Deutsch lernenden Schüler eine bessere Leistung erbrachten, besonders groß war der Unterschied in der NémetvölgyiSchule: Dort konnten zwei Drittel $(66,7 \%)$ der Deutsch lernenden Schüler eine richtige Antwort geben, von den kein Deutsch lernenden Schülern hingegen konnten nur 31 Prozent $(24 \%+7 \%)$ drei oder mehr Länder mit deutscher Amtssprache bezeichnen. 55 Prozent $(35 \%+20 \%)$ der befragten Schüler konnten ein oder mehrere von Ungarndeutschen bewohnte Gebiete angeben. Generell gilt auch hier, dass die Deutschlernenden besser abschneiden, merkwürdigerweise konnten aber viele Schüler (41\%) in der Németvölgyi-Schule, die kein Deutsch lernen, mehrere Gebiete richtig angeben, bei den Deutschlernenden betrug dieser Anteil nur 11 Prozent.

Generell gilt, dass die Mehrheit der befragten Schüler die Zahl der Ungarndeutschen der Kategorie 15.000 zuordnet und damit niedriger einschätzt als in den offiziellen Statistiken angegeben wird (220.000). 
Tabelle 1

Wo leben deutschsprachige Menschen in Europa?

\begin{tabular}{|c|c|c|c|c|c|}
\hline Schule & & $\begin{array}{l}\text { Drei deutsch- } \\
\text { sprachige } \\
\text { Länder richtig }\end{array}$ & $\begin{array}{c}\text { Mehr als } 3 \\
\text { deutschsprachige } \\
\text { Länder richtig }\end{array}$ & $\begin{array}{c}\text { Mangelhaft } \\
\text { oder keine } \\
\text { Antwort }\end{array}$ & $\begin{array}{l}\text { Ins- } \\
\text { gesamt }\end{array}$ \\
\hline \multirow{2}{*}{$\begin{array}{l}\text { Kodály-Schule } \\
\text { in Kecskemét }\end{array}$} & $\begin{array}{l}\text { Lernt } \\
\text { Deutsch }\end{array}$ & $\begin{array}{c}6 \\
30 \%\end{array}$ & $\begin{array}{c}4 \\
20 \%\end{array}$ & $\begin{array}{c}10 \\
50 \%\end{array}$ & $\begin{array}{c}20 \\
100 \%\end{array}$ \\
\hline & $\begin{array}{l}\text { Lernt kein } \\
\text { Deutsch }\end{array}$ & $\begin{array}{c}9 \\
41 \%\end{array}$ & $\begin{array}{c}1 \\
4,5 \%\end{array}$ & $\begin{array}{c}12 \\
54,5 \%\end{array}$ & $\begin{array}{c}22 \\
100 \%\end{array}$ \\
\hline \multirow{2}{*}{$\begin{array}{l}\text { Lónyai-Gym- } \\
\text { nasium in Bu- } \\
\text { dapest }\end{array}$} & $\begin{array}{l}\text { Lernt } \\
\text { Deutsch }\end{array}$ & $\begin{array}{c}12 \\
48 \%\end{array}$ & $\begin{array}{c}1 \\
4 \%\end{array}$ & $\begin{array}{c}12 \\
48 \%\end{array}$ & $\begin{array}{c}25 \\
100 \%\end{array}$ \\
\hline & $\begin{array}{l}\text { Lernt kein } \\
\text { Deutsch }\end{array}$ & $\begin{array}{c}11 \\
39 \%\end{array}$ & - & $\begin{array}{c}17 \\
61 \%\end{array}$ & $\begin{array}{c}28 \\
100 \%\end{array}$ \\
\hline \multirow{2}{*}{$\begin{array}{l}\text { Németvölgyi- } \\
\text { Schule in Bu- } \\
\text { dapest }\end{array}$} & $\begin{array}{l}\text { Lernt } \\
\text { Deutsch }\end{array}$ & $\begin{array}{c}6 \\
66,7 \%\end{array}$ & - & $\begin{array}{c}3 \\
33,3 \%\end{array}$ & $\begin{array}{c}9 \\
100 \%\end{array}$ \\
\hline & $\begin{array}{l}\text { Lernt kein } \\
\text { Deutsch }\end{array}$ & $\begin{array}{c}7 \\
24 \%\end{array}$ & $\begin{array}{c}2 \\
7 \%\end{array}$ & $\begin{array}{c}20 \\
69 \%\end{array}$ & $\begin{array}{c}29 \\
100 \%\end{array}$ \\
\hline Insgesamt & & $\begin{array}{c}51 \\
38 \%\end{array}$ & $\begin{array}{c}8 \\
6 \%\end{array}$ & $\begin{array}{c}74 \\
56 \%\end{array}$ & $\begin{array}{c}133 \\
100 \%\end{array}$ \\
\hline
\end{tabular}

Den Antworten zufolge liegen die Informationsquellen der befragten Schüler mehrheitlich im außerschulischen Bereich (29\% Großeltern, 38\% Fernsehen, Radio). Die Unterschätzung der Anzahl der Ungarndeutschen kann auch damit erklärt werden, dass die Informationen der Kinder über diese nationale Minderheit sich nicht zu einem einheitlichen Bild zusammenformieren. Sie lernen im Geschichtsunterricht, dass im 18. Jahrhundert die deutschsprachige Bevölkerung vorwiegend aus den Gebieten des heutigen Bayern und Baden-Württemberg in Ungarn angesiedelt wurde, um den nach der Türkenherrschaft entstandenen Arbeitskräftemangel zu mildern. Sie haben vielleicht auch darüber gehört, dass viele Deutschsprachige nach dem zweiten Weltkrieg zwangsevakuiert und nach Deutschland ausgesiedelt wurden. Bei Ausflügen und Reisen sehen sie zweisprachige Ortstafeln und Schilder an öffentlichen Gebäuden. Sie hören aber in den ehemaligen „schwäbischen“ Dörfern kaum mehr ein deutsches Wort.

Die Generation der Ungarndeutschen, die noch in allen Lebensbereichen die Muttersprache ihrer Ahnen benutzt hat, ist ausgestorben bzw. alt und zahlenmäßig sehr gering vertreten. Auch die heutigen Eltern in den ungarndeutschen Dörfern können meistens die alte Mundart nicht, und sind deshalb auch nicht in der Lage, diese ihren Kindern weiterzugeben. Selbst wenn die Wiederbelebung der Minderheitenkultur heutzutage auch von der Großpolitik unterstützt wird, geht es eher um die Pflege einer 
Tabelle 2

Wo leben Angehörige der deutschen Nationalität in Ungarn (Ungarndeutsche)?

\begin{tabular}{llccccc}
\hline Schule & & $\begin{array}{c}\text { l Gebiet } \\
\text { richtig }\end{array}$ & $\begin{array}{c}\text { Mehrere } \\
\text { richtig }\end{array}$ & $\begin{array}{c}\text { Richtig } \\
\text { +falsch }\end{array}$ & $\begin{array}{c}\text { Falsch/keine } \\
\text { Antwort }\end{array}$ & $\begin{array}{c}\text { Ins- } \\
\text { gesamt }\end{array}$ \\
\hline \multirow{3}{*}{$\begin{array}{l}\text { Kodály-Schule } \\
\text { in Kecskemét }\end{array}$} & $\begin{array}{l}\text { Leutsch } \\
\text { Lernt kein }\end{array}$ & $45 \%$ & $10 \%$ & $10 \%$ & $35 \%$ & $100 \%$ \\
& Deutsch & $13,6 \%$ & $4,5 \%$ & $31,9 \%$ & $50 \%$ & $100 \%$ \\
Lónyai-Gym- & Lernt & 8 & 6 & 2 & 7 & 20 \\
nasium in Bu- & Deutsch & $32 \%$ & $24 \%$ & $16 \%$ & $28 \%$ & $100 \%$ \\
dapest & Lernt kein & 11 & 4 & 4 & 9 & 28 \\
& Deutsch & $39 \%$ & $14 \%$ & $14 \%$ & $33 \%$ & $100 \%$ \\
Németvölgyi- & Lernt & 6 & 1 & 2 & & 9 \\
Schule in Bu- & Deutsch & $67 \%$ & $11 \%$ & $22 \%$ & & $100 \%$ \\
dapest & Lernt kein & 9 & 12 & 4 & 4 & 29 \\
& Deutsch & $31 \%$ & $41 \%$ & $14 \%$ & $14 \%$ & $100 \%$ \\
Insgesamt & & 46 & 26 & 23 & 38 & 133 \\
& & $35 \%$ & $20 \%$ & $17 \%$ & $28 \%$ & $100 \%$ \\
\hline
\end{tabular}

Tabelle 3

Wie viele Angehörige der deutschen Minderheit (Ungarndeutsche) leben in Ungarn?

\begin{tabular}{|c|c|c|c|c|c|}
\hline Schule & & 1.000 & 15.000 & 220.000 & Insgesamt \\
\hline \multirow{4}{*}{$\begin{array}{l}\text { Kodály-Schule } \\
\text { in Kecskemét }\end{array}$} & Lernt & 4 & 16 & \multirow[t]{2}{*}{-} & 20 \\
\hline & Deutsch & $20 \%$ & $80 \%$ & & $100 \%$ \\
\hline & Lernt kein & 1 & 19 & 2 & 22 \\
\hline & Deutsch & $5 \%$ & $86 \%$ & $9 \%$ & $100 \%$ \\
\hline \multirow{4}{*}{$\begin{array}{l}\text { Lónyai-Gym- } \\
\text { nasium in Bu- } \\
\text { dapest }\end{array}$} & Lernt & \multirow[t]{2}{*}{-} & 16 & 8 & 25 \\
\hline & Deutsch & & $64 \%$ & $32 \%$ & $100 \%$ \\
\hline & Lernt kein & 5 & 16 & 7 & 28 \\
\hline & Deutsch & $18 \%$ & $57 \%$ & $25 \%$ & $100 \%$ \\
\hline \multirow{4}{*}{$\begin{array}{l}\text { Németvölgyi- } \\
\text { Schule in Bu- } \\
\text { dapest }\end{array}$} & Lernt & \multirow[t]{2}{*}{-} & 8 & 1 & 9 \\
\hline & Deutsch & & $89 \%$ & $11 \%$ & $100 \%$ \\
\hline & Lernt kein & 1 & 14 & 14 & 29 \\
\hline & Deutsch & $3,4 \%$ & $48,3 \%$ & $48,3 \%$ & $100 \%$ \\
\hline
\end{tabular}


DAS BILD UNGARISCHER SCHÜLER VON DEN DEUTSCHSPRACHIGEN LÄNDERN 215

Tabelle 4

Informationsquellen über Ungarndeutsche

\begin{tabular}{lcc}
\hline Quelle & Gewählt wurde & Anteil \% \\
\hline Großeltern & 61 & 29 \\
Schule & 51 & 24 \\
Freunde & 5 & 3 \\
Fernsehen, Radio, Zeitung & 80 & 38 \\
Sonstige & 13 & 6 \\
\hline
\end{tabular}

Tabelle 5

Angaben in der Reihenfolge, wie oft die Begriffe insgesamt an einer der ersten drei Stellen genannt wurden

\begin{tabular}{lcccc}
\hline $\begin{array}{l}\text { Die zur Wahl gestellten } \\
\text { Angaben in Häufigkeitsfolge }\end{array}$ & $\begin{array}{c}1 . \\
\text { Stelle }\end{array}$ & $\begin{array}{c}2 . \\
\text { Stelle }\end{array}$ & $\begin{array}{c}3 . \\
\text { Stelle }\end{array}$ & $\begin{array}{c}\text { Insgesamt an einer der } \\
\text { ersten 3 Stellen gewählt }\end{array}$ \\
\hline Autos & 22 & 27 & 31 & 80 \\
Fußball & 28 & 23 & 17 & 68 \\
Komponisten & 12 & 16 & 19 & 47 \\
der zweite Weltkrieg & 19 & 13 & 3 & 36 \\
Freunde, Bekannte & 19 & 13 & 3 & 35 \\
Sonstige & 18 & 6 & 4 & 28 \\
Dichter, Schriftsteller & 2 & 6 & 2 & 10 \\
\hline
\end{tabular}

Tabelle 6

Häufigkeit der Informationsquellen über Deutsche

\begin{tabular}{lcc}
\hline Quelle & Häufigkeit & Zahl des Vorkommens \\
\hline In der Schule & $29 \%$ & 62 \\
Zu Hause & $25 \%$ & 54 \\
Filme & $24 \%$ & 52 \\
Bücher & $13 \%$ & 26 \\
Anderswo & $9 \%$ & 17 \\
\hline
\end{tabular}


Tabelle 7

Zuordnung von Eigenschaften (Deutsche-Ungarn)

\begin{tabular}{cccccc}
\hline $\begin{array}{c}\text { Ungarn } \\
\%\end{array}$ & $\begin{array}{c}\text { Deutsche } \\
\%\end{array}$ & $\begin{array}{c}\text { Eigenschaft } \\
\text { (Positiv) }\end{array}$ & $\begin{array}{c}\text { Eigenschaft } \\
\text { (Negativ) }\end{array}$ & $\begin{array}{c}\text { Deutsche } \\
\%\end{array}$ & $\begin{array}{c}\text { Ungarn } \\
\%\end{array}$ \\
\hline 58 & 44 & beweglich & schwerfällig & 45 & 29 \\
46 & 63 & fleißig & faul & 34 & 44 \\
40 & 12 & bescheiden & anmaßend & 68 & 44 \\
66 & 58 & tapfer, mutig & feige, ängstlich & 16 & 19 \\
52 & 41 & tolerant & intolerant & 39 & 22 \\
61 & 59 & phantasievoll & phantasielos & 30 & 21 \\
42 & 41 & großzügig & kleinlich & 35 & 39 \\
82 & 29 & geistreich & humorlos & 49 & 12 \\
51 & 40 & aufrichtig & falsch & 33 & 31 \\
51 & 37 & friedlich & streitsüchtig & 50 & 31 \\
50 & 79 & kultiviert & primitiv & 10 & 30 \\
76 & 31 & warmherzig & kalt & 38 & 9 \\
\hline
\end{tabular}

Tabelle 8

Berühmte Deutsche

(Zahl der abweichenden Antworten)

\begin{tabular}{|c|c|c|c|c|c|}
\hline Berühmte Deutsche & Musik & Literatur & Philosophie & Geschichte & Sonstige \\
\hline Bach & $\begin{array}{l}\text { Typische } \\
\text { Antwort }\end{array}$ & & & & 1 \\
\hline Beethoven & $\begin{array}{l}\text { Typische } \\
\text { Antwort }\end{array}$ & & & & \\
\hline Goethe & 1 & $\begin{array}{l}\text { Typische } \\
\text { Antwort }\end{array}$ & 26 & 1 & \\
\hline Brüder Grimm & & $\begin{array}{l}\text { Typische } \\
\text { Antwort }\end{array}$ & 2 & 6 & \\
\hline Hitler & & 3 & & $\begin{array}{l}\text { Typische } \\
\text { Antwort }\end{array}$ & $\begin{array}{l}\text { Religions- } \\
\text { kunde (2) }\end{array}$ \\
\hline Luther & & & & $\begin{array}{l}\text { Typische } \\
\text { Antwort }\end{array}$ & $\begin{array}{l}\text { Religions- } \\
\text { kunde (4) }\end{array}$ \\
\hline Thomas Mann & 1 & $\begin{array}{l}\text { Typische } \\
\text { Antwort }\end{array}$ & 31 & 3 & \\
\hline
\end{tabular}

EJMH 5, 2010 
Tradition, während das Alltagsleben in ungarischer Sprache geführt wird. Dies bereitet auch den Jugendlichen ungarndeutscher Abstammung Identitätsprobleme und macht zugleich die Anwesenheit dieser Volksgruppe für die andere Bevölkerung wenig transparent. Diese Erfahrungen habe ich vor Jahren als Lehrer in einer so genannten Nationalitätenschule in Budaörs gesammelt.

Generell gilt, dass der eigenen Nation mehr positive und umgekehrt den Deutschen mehr negative Eigenschaften zugeordnet wurden. Eine Ausnahme bildet das Eigenschaftspaar fleißig - faul.

Merkwürdig viele, das heißt 26 bzw. 31 Schüler stimmten bei Goethe und Thomas Mann für Philosophie.

Frage 7.: Warst du schon mal in einem deutschsprachigen Land?

$\begin{array}{lrr}\text { Noch nie: } & 32 & 24 \% \\ \text { Deutschland: } & 79 & 59 \% \\ \text { Österreich: } & 25 & 19 \% \\ \text { Schweiz: } & 3 & 2 \%\end{array}$

Frage 8.: In welches würdest du am liebsten reisen, wenn du die Möglichkeit hättest?

$\begin{array}{lr}\text { Ablehnung: } & 8 \\ \text { Deutschland: } & 45 \\ \text { Österreich: } & 32 \\ \text { Schweiz: } & 42\end{array}$

Sonstige Antworten: Großbritannien, Paris, die Niederlande

Es ist festzustellen, dass die Schweiz den Schülern am wenigsten bekannt ist, zugleich aber eine starke Anziehungskraft ausübt. Die wenigsten waren dort persönlich, die meisten haben auf der Blindkarte die Schweiz nicht als deutschsprachiges Land einzeichnen können. Trotzdem ist ihr Bild über dieses Alpenland eindeutig positiv. Als Ursache werden am häufigsten die Landschaft, die guten Skifahrtmöglichkeiten, die Schweizer Schokolade, die Ordnung und der Wohlstand erwähnt.

Aus mehreren Antworten wird deutlich, dass eine kleine, aber feste Minderheit unter den Schülern - und das trifft auf alle drei untersuchten Schulen zu - die Deutschen und ihr Land strikt ablehnt. Es kamen Antworten, wie „Deutsche gibt es genügend auch am Balaton, ich brauche nicht hinzureisen“, „Ich hasse die Deutschen“, oder „Sie ekeln mich an“. Die meisten negativen Erfahrungen haben die Kinder anscheinend im Kontakt mit deutschen Touristen in Ungarn gesammelt. Deutsche als Gastgeber in ihrer Heimat wurden eher gelobt.

Frage 9.: Warum lernst du Deutsch?

- Ich mag die Deutschen / Österreicher. (5)

- Ich möchte mehrmals in deutsches Sprachgebiet reisen, vielleicht auch dort lernen, arbeiten. (18)

- Es war die Wahl meiner Eltern / Familie. (20)

- In der Schule konnte nur diese Sprache gelernt werden. (1)

- Ich wurde einer Deutsch lernenden Gruppe zugeteilt, und ich freue mich darüber. (3) 
Tabelle 9

Wie viele Schüler lernen Deutsch?

\begin{tabular}{|c|c|c|c|c|}
\hline Schule & Lernt Deutsch & & \multicolumn{2}{|c|}{ Lernt kein Deutsch } \\
\hline Lónyai in Budapest & 25 & & 28 & \\
\hline Kodály in Kecskemét & 20 & & 22 & \\
\hline Németvölgyi in Budapest & 9 & & 29 & \\
\hline Insgesamt & 54 & $40 \%$ & 79 & $60 \%$ \\
\hline
\end{tabular}

Lehrbücher: Schrittweise, Sowieso, Start, Deutschmobil

- Ich wurde einer Deutsch lernenden Gruppe zugeteilt, obwohl ich lieber eine andere Sprache hätte lernen wollen. (16)

Wenn ja, welche? (lieber Englisch, Italienisch)

Frage 10.: Kann jemand bei Euch Deutsch? Unterstreiche die Antwort!

\begin{tabular}{|c|c|}
\hline Großeltern & \\
\hline Eltern & \\
\hline Geschwister & \\
\hline Sonstige & \\
\hline Beruf der Eltern: & \\
\hline Unternehmer & \\
\hline Akademiker & \\
\hline Angestellter & \\
\hline Arbeiter & \\
\hline Hausfrau & \\
\hline
\end{tabular}

Die berufliche Zuordnung der Eltern zu einzelnen Kategorien war manchmal nicht einfach, da Antworten, wie „Er / Sie arbeitet bei einer Firma“ oder „Sie arbeitet aus familiären Gründen momentan nicht" schwer zu deuten waren.

\subsection{Die Interpretation der Ergebnisse}

Wenn man berücksichtigt, dass die für die Erhebung ausgewählten Schulen sowohl hinsichtlich des Unterrichtsniveaus, als auch der von zu Hause mitgebrachten Kenntnisse der Schüler über dem Durchschnitt stehen, sind die Ergebnisse nicht sehr erfreulich. Besonders trifft das auf die Lösungen der Fragen nach geographischen Kenntnissen zu.

Insgesamt 44 Prozent der befragten Schüler haben auf der Landkartenskizze drei oder mehr von Deutschsprachigen bewohnte Gebiete eingezeichnet. 66 Prozent der Schüler haben die Aufgabe mangelhaft gelöst. Die meisten Fehler haben sich dadurch 
ergeben, dass die Schweiz als deutschsprachiges Land nicht berücksichtigt wurde. Ein anderer Mangel war es, dass BENELUX-Staaten für komplett deutschsprachig gehalten wurden. Nur acht Schüler konnten - außer Deutschland, Österreich und der Schweiz - solche Gebiete in Europa angeben, in denen eine deutschsprachige nationale Minderheit lebt. Von ihnen haben fünf Kinder Deutschunterricht in der Schule. Obwohl die Schüler, die in der Schule Deutschunterricht haben, mit 53 Prozent der richtigen Antworten (drei oder mehr Länder mit deutscher Bevölkerung richtig markiert) über dem Durchschnitt lagen, zeigt dieser Anteil, dass der Deutschunterricht derartige Geographiekenntnisse der Schüler wenig beeinflusst hat.

Von den durch Ungarndeutsche bewohnten Gebieten sind Ödenburg (Sopron) und Umgebung sowie allgemein das Grenzgebiet in Westungarn am meisten bekannt. Danach folgen die Umgebung von Budapest und das Komitat Baranya. Die Schüler der Kodály-Schule in Kecskemét haben häufiger die von Ungarndeutschen bewohnten Gebiete im Komitat Bács-Kiskun eingezeichnet.

Die meisten Schüler (76\%) unterschätzen die Anzahl der Ungarndeutschen, die statistischen Daten zufolge bei 220.000 liegt. Unter den 32 Schülern, die eine richtige Antwort gegeben haben, konnte keine Korrelation zwischen Deutschunterricht und Kenntnissen festgestellt werden. Unter den Schülern, die kein Deutsch lernen, haben sogar mehr die Anzahl der Ungarndeutschen richtig eingeschätzt, als bei den Deutschlernenden.

Unter den Informationsquellen über Ungarndeutsche spielen die Massenmedien (Fernsehen, Rundfunk und Zeitung) mit 38 Prozent eine bedeutende Rolle. Danach folgen die Eltern und Großeltern mit 29 Prozent sowie die Schule (24\%).

Die Antworten auf die Frage 3. zeigen, dass die Schüler beim Wort „deutsch“ eher mit positiven Errungenschaften der Deutschen (Autos, Fußball, Komponisten) assoziieren, dass sie ihre Informationen und Eindrücke über die Deutschen vorwiegend in der Schule, in der Familie bzw. aus Filmen gewinnen und dass sie die Deutschen mehrheitlich für fleißige, tapfere, ideenreiche, gebildete, aber auch streitsüchtige Menschen halten. Insgesamt wurden aber den Ungarn viel mehr positive Eigenschaften zugeordnet, als den Deutschen. In diesem Zusammenhang gab es keinen Unterschied zwischen den Schülern, die Deutsch lernen und denen, die keinen Deutschunterricht in der Schule haben. Das ist ein Beispiel dafür, dass bei Vorurteilen häufig längst vergangene historische Ereignisse eine besondere Rolle spielen, indem ,in ihnen hervorgetretene Merkmale, die an eine ganz bestimmte Situation gebunden sind, als Dauermerkmale ausgegeben werden“ (KÖNIG 1971, 73).

Bei den frei ergänzbaren Stichwörtern der Frage 3. wurden bei der Wahlmöglichkeit „sonstige“ überwiegend positive oder eher positiv deutbare Dinge erwähnt (Deutschstunde, Bier, Familienurlaub, Verwandte, deutsche Speisen, Sprachen lernen, schöne Landschaft, Spiele, Luther, Deutschland ist schön, sauber und diszipliniert, die deutsche Wirtschaft, präzise, Städte, entwickeltes, ordentliches Land, lieben die Ordnung, Wohlstand). Auf den Abbau der Stereotypen deutet, dass viele der befragten Schüler sich hinsichtlich mehrerer, in Frage 5. erwähnter Eigenschaften nicht entscheiden konnten. 
In diesem Zusammenhang spielt auch das Spiegelbild des nationalen Selbstbewusstseins eine Rolle. Die Einstellung zu Ausländern wird sowohl bei den Kindern, als auch bei den Erwachsenen stark von dem eigenen Selbstgefühl als Ungar beeinflusst (PETNEKI 1994, 123).

Bei der Analyse der Antworten auf die Frage 6. konnte man feststellen, dass eine große Mehrheit der Schüler die angegebenen berühmten Deutschen richtig kannte und der entsprechenden Disziplin zuordnen konnte. Die größten Abweichungen gab es bei Luther und Thomas Mann. Luther wurde am häufigsten der Geschichte zugeordnet, aber Literatur, Philosophie und Religionsunterricht, die ebenfalls als richtige Antworten angesehen werden können, kamen auch öfter vor. Neun Schüler haben Goethe fälschlicherweise der Musik zugeordnet. 26 Schüler, also etwa 20 Prozent aller Befragten haben Goethe der Philosophie zugeordnet und etwa ein Viertel hat auch Thomas Mann dazu gerechnet. Eine Erklärung dafür kann sein, dass beide Personen nicht zum Grundlehrstoff der allgemeinbildenden Schule gehören, und im Unterricht nur nebenbei erwähnt werden. 2005, das Jahr, in dem diese Erhebung durchgeführt wurde, ist zugleich das Jubiläumsjahr von Attila József. Am ehesten können die Schüler den Namen Thomas Manns im Zusammenhang mit seiner Begegnung mit Attila József kennen, die in der ungarischen Literaturstunde behandelt wird.

Etwa zwei Drittel aller Befragten behaupteten, in einem der deutschsprachigen Länder gewesen zu sein. Die meisten waren in Deutschland. Es ist also anzunehmen, dass auch persönliche Eindrücke bei der Prägung ihres Deutschbildes eine Rolle spielen. Zwar kann man zwischen der Zuordnung von Eigenschaften (Frage 5.), wo es für die Deutschen eher negative Merkmale gab, und der Frage 3. („Was fällt dir spontan ein, wenn du das Wort 'deutsch' hörst?"), wo in der Mehrheit positiv gewählt wurde, gewisse Ambivalenzen bei den Schülern feststellen. Ebenfalls zeigen sich Widersprüche zwischen den Antworten zu Frage 5. und den Begründungen bei Frage 8. (,Wo würdest du hinfahren?"“), wo über Deutschland, das Wunschziel Nr. 1 mehrheitlich sehr positiv geurteilt wird. Außer der Neugier, ,,ich möchte Deutschland kennen lernen“, wurden Gründe geschrieben, wie „gute Schokolade“, „,dort leben meine Pateneltern“, „Verwandte leben dort, schönes Land“, „schönes, modernes Land“, „gute Gastfreundschaft, schöne Landschaft“ „Münchner Fußballspieler“, „, das Leben ist dort leichter, und die Menschen sind freundlicher“, „,sauberes Land, eine interessante Ausstellung zieht mich hin“, „eines meiner Lieblingsländer“, „,sehr entwickeltes Land“, „berühmtes Land“ „,nach Berlin, dort könnte ich die deutsche Sprache üben“, „menschenfreundliche, gebildete Menschen leben dort“ oder „Freundin lebt dort“. Diejenigen, die mit „nein“ geantwortet haben, lehnen gleichermaßen alle deutschsprachige Länder markant ab. Gründe: ,ich hasse die Deutschen und mag auch die Sprache nicht“, „Deutsche gibt es genügend auch am Plattensee“, ,ich mag die Deutschen einfach nicht" usw. Bei Österreich und der Schweiz als Wunschziel wurden unter anderem die schöne Landschaft und die guten Möglichkeiten zum Skifahren hervorgehoben, oder es wurden die Schweizer Schokolade, die Stadt Wien bzw. Verwandte erwähnt.

Die Antworten auf die Frage 9. (,Warum lernst du Deutsch?“) zeigen eindeutig, dass diese Sprache auch unter den Deutsch lernenden Schülern nicht besonders „in“ ist. Viele Antworten deuten darauf hin, dass eher äußere Zwänge dazu führten, dass 
die Kinder gerade diese Sprache lernen. In den untersuchten drei Schulen nehmen insgesamt 54 Schüler am Deutschunterricht teil. Zwei Drittel von ihnen haben sich so geäußert, dass diese Sprache entweder die Wahl ihrer Eltern / Familie war, oder dass sie in der Schule einer Klasse mit Deutschunterricht zugeteilt wurden, obwohl sie lieber eine andere Sprache (in der Mehrheit Englisch oder Italienisch) hätten lernen wollen. Fast ein Drittel aller Deutschlernenden hätte eine andere Sprache gewählt. Ein Drittel der Antwortgeber hat sich so geäußert, dass sie spätere Ziele mit dieser Sprache hätten. Nur eine Minderheit hat sich eindeutig positiv geäußert. Besonders diejenigen, die offensichtlich noch nicht einsehen konnten, dass Fremdsprachenkenntnisse neue Möglichkeiten eröffnen und auch im Privatleben gut zu gebrauchen sind, finden, dass sich die Anstrengung, Deutsch zu lernen, nicht lohne. In der Regel würden sie stattdessen Englisch lernen, weil das stärker im Trend ist und scheinbar leichter erlernt werden kann.

Die letzte und abschließende Frage ergab, dass das Deutsch-Können immer noch eine Tradition in Ungarn zu haben scheint. 70 Prozent aller befragten Schüler gaben an, dass jemand in ihrer Familie Deutsch könne.

\section{Vergleich der Ergebnisse von 1980 mit denen von 2005}

Ein leichter, 6-prozentiger Rückgang gegenüber 1980 ist bei den Schülern festzustellen, die alle deutschsprachigen Länder auf der Landkarte richtig markiert haben. Nur 6 Prozent der Schüler haben außer den drei Ländern (Deutschland, Österreich und Schweiz) auch weitere Gebiete angezeichnet, wo eine deutschsprachige Minderheit lebt. Die meisten von den insgesamt 8 Antwortgebern, die zu dieser Gruppe gehören, haben Teile der BENELUX-Staaten, die ehemaligen sudetendeutschen Gebiete in Tschechien oder auch das französische Elsass-Lothringen angegeben, keiner aber SüdTirol in Norditalien, das jedoch vor 25 Jahren mehrmals vorkam. Generell gesehen ist die Verschlechterung der geographischen Kenntnisse über die deutschsprachigen Gebiete meiner Meinung nach auch damit zu erklären, dass die besser gewordenen Reisemöglichkeiten das Interesse an theoretischen Kenntnissen in Geographie zurückgedrängt haben, und dass die Informationen der Schüler über fremde Länder eher durch persönliche Erfahrungen oder aus den Medien gewonnen werden, in denen zum Beispiel von Süd-Tirol kaum die Rede ist und das auch nicht zu den frequentierten Reisezielen der Ungarn gehört. Ein Widerspruch war dabei festzustellen, dass viele der befragten Schüler gerne in die Schweiz reisen würden - wie sie sagten, wegen der schönen Landschaft und der guten Skifahrtmöglichkeiten -, dieselben waren aber zugleich nicht in der Lage, die Schweiz als deutschsprachiges Land auf der Blindkarte einzuzeichnen.

Wie vor 25 Jahren unterschätzen die meisten Schüler (76\%) die Anzahl der Ungarndeutschen (,Schwaben“). In dieser Hinsicht ist ein 13-prozentiger Rückgang festzustellen. Bei der örtlichen Bestimmung der von Ungarndeutschen bewohnten Gebiete waren den Befragten diejenigen Gebiete am häufigsten eingefallen, die am nahesten zu ihrem Wohnort liegen. Mehrere Schüler haben in allen drei Schulen fälschlicher- 
weise die Ortschaften am Balaton (Plattensee) als von Deutschen bewohnt bestimmt. Es war für sie offensichtlich schwierig, die Ferienorte mit deutschen Touristen von den ungarndeutschen Dörfern zu unterscheiden. Was die Informationsquellen über Deutschstämmige in Ungarn betrifft, wurden, wie auch vor 25 Jahren, am häufigsten die Medien angegeben, danach folgten, ebenfalls wie früher, die Eltern und Großeltern. Der Anteil der letzteren ist aber leicht zurückgegangen.

Eine Verschiebung der Eindrücke über die Deutschen zeigten die Antworten auf die Fragen 3. bis 5. Die Erhebung im Jahre 1980 hatte gezeigt, dass damals das Deutschbild der Schüler überwiegend durch Kriegsfilme über den zweiten Weltkrieg beeinflusst war. Obwohl dieses Motiv sich auch 2005 als wichtig erwies, dominierten andere Aspekte davor. Das ist unter anderem mit der politischen Wende vor 15 Jahren und mit dem Rückgang der damals noch ziemlich verbreiteten Einstellung, derzufolge die Deutschen mit den Kriegsverbrechen des Nazi-Deutschlands gleichgesetzt wurden, zu erklären. Für die Mehrheit der Schüler sind heutzutage für die Bildung ihrer Meinung über die Deutschen Industrieprodukte - wie etwa Autos -, Sporterfolge - wie zum Beispiel beim Fußball - sowie persönliche Begegnungen scheinbar wichtiger geworden. Dies ist unter anderem auch mit den schon erwähnten besseren Reisemöglichkeiten und damit auch mit den differenzierteren eigenen Erfahrungen zu erklären. Als wichtigste Quellen für ihre Erfahrungen mit Deutschen wurden von den befragten Schülern vorwiegend die Schule und das Elternhaus genannt. Bei der Frage 5., wo man zutreffende Eigenschaften wählen sollte, zeigten sich - wie auch früher - verbreitete Stereotypen; die Anzahl der Antwortgeber, die bei bestimmten Eigenschaftspaaren nicht wählen konnten oder wollten, hat sich erhöht. Dies deutet meiner Meinung nach darauf hin, dass die Kinder auch durch das demokratischer gewordene öffentliche Denken kritischer und vorsichtiger bei ihren Urteilen geworden sind und sich von verbreiteten Vorurteilen über eine andere Nation etwas weniger verleiten lassen. Vor 25 Jahren war es mit den Kriegsfilm-Vorurteilen zu erklären, dass die Meinungen beim Eigenschaftspaar „fleißig - faul“ 50 Prozent zu 50 Prozent pro und kontra waren, obwohl die Deutschen im allgemeinen Bewusstsein als fleißiges Volk gelten. In der neuen Erhebung vom Jahre 2005 haben sich 63 Prozent der Befragten für „fleißig“ entschieden. Ansonsten wurden den Deutschen immer noch eher negative Eigenschaften zugesprochen, und beide Nationen wurden mehrheitlich so beurteilt, wie vor einem Vierteljahrhundert. Einzig in der Frage der Kultiviertheit wurde den Deutschen eine viel größere Überlegenheit zugesprochen, als den Ungarn. Die Urteile bzw. Vorurteile haben sich weiter gehalten, obwohl sich die Schwergewichte bei den zur Verfügung stehenden Informationen und den zu Grunde liegenden Eindrücken geändert haben müssen.

Bei der Frage 6., wo berühmte Deutsche einzelnen Disziplinen zugeordnet werden sollten, gab es, wie auch vor 25 Jahren, mit der Bestimmung der beiden Komponisten Bach und Beethoven die wenigsten Probleme. Eine positive Verschiebung war bei der Bekanntheit von Martin Luther festzustellen. Dies ist unter anderem mit der politischen Wende 1989-1990 und der damit verbundenen anderen Einschätzung der Religionsfragen zu erklären. Der Reformator Luther ist zwar unter den Schülern wesentlich bekannter geworden, wurde aber mit großer Streuung unterschiedlichen Disziplinen (mehrheitlich Geschichte, aber auch Literatur oder Philosophie bzw. Reli- 
gionsunterricht) zugeschrieben. Diese Zuordnungen können nicht als falsch aufgefasst werden, zeigen aber, dass es immer noch eine gewisse Unsicherheit unter den Schülern bezüglich Luthers Person gibt. Bei der Auswertung der Antworten zu den Komponisten und zu Luther muss man auch festhalten, dass die eine Schule in Kecskemét, wo die Erhebung durchgeführt wurde, eine Musikschule, das Lónyay-Gymnasium aber eine kirchliche Schule mit sechs Klassenstufen ist.

Eine große Veränderung war bei der Frage 7. (,Warst du schon mal in einem deutschsprachigen Land; wenn ja, wo?“) festzustellen. Im Jahre 1980 hatte ein Drittel der Antwortgeber erklärt, schon in einem deutschsprachigen Land gewesen zu sein. 2005 stieg dieser Anteil auf 76 Prozent. Die bedeutende quantitative Änderung ist mit den inzwischen verbesserten, freien Reisemöglichkeiten zu erklären. Gegebene Antworten auf andere Fragen des Fragebogens verweisen aber darauf, dass viele der Schüler auf einer Skireise oder zu Verwandtenbesuch in einem deutschsprachigen Land waren, und dass deshalb diese Aufenthalte nur begrenzt zu dem direkten Kontakt mit der Bevölkerung beitrugen.

Bei der Frage 8. (,Wo würdest du hinfahren?“) hat sich die Anziehungskraft der Landschaft Österreichs und der Schweiz kaum geändert. Vor 25 Jahren fühlten sich die meisten Schüler - neben Familienbesuchen - von technischen Konsumgütern in den westlichen Teil des damals geteilten Deutschlands angezogen. 2005 wurde auch hier neben dem Besuch von Verwandten die Landschaft als Reiseursache genannt. Viele, vorwiegend Jungen möchten den deutschen Fußball vor Ort erleben. Auch bei dieser Frage meldeten sich, ähnlich wie früher (1980) geographische Unsicherheiten, bei denen Großbritannien, Paris oder die BENELUX-Staaten für deutschsprachig gehalten wurden.

Frage 9.: Es gab im Jahre 2005 in den untersuchten Klassen viel mehr Schüler (40\%), die Deutsch lernen, als 1980. Bei der früheren Erhebung gab es nur eine Klasse mit so genanntem erweiterten Deutschunterricht, die damals als Kontrollklasse diente, und nur fast 20 Prozent aller befragten Schüler ausmachte. Wenn man bedenkt, dass die Eltern der kürzlich befragten Schüler die Generation der im Jahre 1980 durchgeführten Erhebung bildeten, und angenommen, dass die Angaben der Antwortgeber stimmen, spiegeln sich heute die damaligen Möglichkeiten zum Deutschlernen wider. Wenn es stimmt, dass 42 Prozent der heutigen Eltern Deutsch können, müsste etwa die Hälfte von ihnen die Sprache schon als Erwachsener gelernt haben.

Man kann feststellen, dass die ungarischen Kinder ihre Informationen über Deutsche aus sehr verschiedenen Quellen gewinnen, dass sich aber diese Informationen oft nicht zu einer kohärenten Einheit in ihrem Kopf zusammenfügen. Sporadische Eindrücke, gelernte Kenntnisse und äußere Einflüsse rufen bestimmte Urteile hervor, die dann parallel nebeneinander wirken. Manche loben die materiellen Werte und die Landschaft der deutschsprachigen Länder, lehnen aber aufgrund von schlechten Erfahrungen - zum Beispiel mit deutschen Touristen am Plattensee - die Deutschen als Menschen ab. Bei manchen wirkt sich das auch auf die Sympathie gegenüber der Sprache als Unterrichtsgegenstand aus. Es hängt sehr vom Zufall ab, welche Eindrücke am meisten dominant sind. Diese Frage berührt auch das Interessenfeld der Stereotypenforschung. 


\section{Stereotypen}

\subsection{Die Stereotypen und die Stereotypenforschung}

- Stereotypen ${ }^{1}$ sind verfestigte kollektive Zuschreibungen mit vorwiegend emotionalem Gehalt, die nur in ihren sprachlichen bzw. bildlichen Repräsentationen zu fassen sind;

- die Stereotypenforschung versucht nicht, den Wahrheitsgehalt von Stereotypen zu ermitteln oder zu widerlegen, sondern ihre Funktion und Wirkung in gesellschaftlichen Diskursen zu erforschen;

- die historische Stereotypenforschung untersucht darüber hinaus die Korrelationen zwischen der Genese, Funktion und Wirkung von Stereotypen in den Prozessen kollektiver Identitätsbildungen;

- die Interdependenz und Interaktion von Autostereotypen und Heterostereotypen gehört zu den bevorzugten Forschungsinteressen der Oldenburger AHS, mit dem Ziel, zur Entwicklung der historischen Multiperspektivität in der Geschichtsschreibung Mitteleuropas beizutragen.

\subsection{Hunyadys Erkenntnisse}

Bei der Herausbildung von Stereotypen über andere Nationen spielen die Stereotypen über die eigene Nation eine wichtige Rolle. Untersuchungsergebnisse, die von György Hunyady zitiert werden, zeigten, dass bei Befragungen viel mehr Ungarn es ablehnten, sich über schlechte Eigenschaften des eigenen Volkes zu äußern, als über die positiven. Das eigene Volk wird im Allgemeinen positiver beurteilt als ein fremdes. Unkenntnis und lückenhafte Informationen ziehen eher ablehnende Haltungen und Vorurteile nach sich (HUNYADY 1996, 107). Hunyady bezieht sich auf Erhebungen in den Jahren 1981 und 1991, wonach bestimmte Verschiebungen bezüglich der Beurteilung der Deutschen festzustellen waren.

Das Bestehen der Bundesrepublik Deutschland und der Deutschen Demokratischen Republik, das heißt, die Teilung des Deutschtums hat 1981 die allgemeine Charakterisierung des deutschen Menschen nicht leichter gemacht. An führender Stelle in den Antworten der befragten Personen standen Patriotismus, Selbstbewusstsein und politisierende Mentalität, gemischt mit intellektuellen Tugenden. Am häufigsten wurde dem deutschen Menschen die Soziabilität abgestritten, die damit verbundenen Eigenschaften, wie Popularität, freundliche Natur und guter Humor lagen hinter den moralischen Zügen.

Die Faktorenstruktur der Charakteristik ist besonders komplex, hier hat man sich 1981 mit vier Faktoren eklatant konfrontiert. Die dominante hintere Variable meldet sich bei den am meisten positiven Zügen, und zwar bei der Beurteilung, ob der

\footnotetext{
${ }^{1}$ www.bohemistik.de/quellentexte.html.
} 
Deutsche patriotisch, vernünftig oder selbstbewusst sei, was auch mit der Frage der Ehrlichkeit verbunden ist. Kein Teil dieses Eigenschaftskomplexes ist aber die politische Betroffenheit, die einen selbstständigen Faktor bildet. Die Faktoranalyse deckt zwei weitere Variablen auf, die eine steckt hinter dem Fleiß und der Bildung, die andere verbindet die verschiedenen Äußerungen der Soziabilität miteinander, denen gegenüber man mit der größten Skepsis stand. Die Faktorenstruktur hat sich im Vergleich zu 1991 nur soweit geändert, dass sich zu den größten Variabeln mit erklärendem Charakter ein anderer Faktor mit weniger Stärke hinzugefügt hat, zum Kreis der zuerst erwähnten Eigenschaften ist also auch die politische Mentalität gekommen. Der innere Aufbau der Charakteristik ist einfacher geworden, Zusammensetzung und Rolle der beiden anderen Faktoren blieben unverändert.

Im Jahre 1991, wo die veränderte politische Situation besser durchschaubar wurde, kam der Charakterzug ,selbstbewusst“ auf den ersten Platz der Eigenschaftsskala für die Charakteristik der Deutschen. Danach drängen sich die zum dominanten Faktor gehörenden Eigenschaften, „vernünftig“ wurde ein wenig zurückgedrängt, „herlich“ ist ein wenig vorwärts gerückt. Die politische Beteiligtheit, das neuere Mitglied des Eigenschaftskomplexes, das eine mildere Rolle als früher beim Gesamtbild der Deutschen spielt, ist etwas zurückgeblieben. Im Mittelfeld nimmt die Beurteilung des Fleißes und der Bildung Platz. Auch die innere Reihenfolge der drei Züge der Soziabilität ist gegenüber früher gleich geblieben. Die Verschiebung des allgemeinen Niveaus der Bewertung war bei der Charakterisierung des „Deutschen“ gering, der Durchschnitt ist im Jahre 1991 von 5,2 auf 4,9 gesunken (HUNYADY 1996, 119-20).

Tabelle 10

Nationalcharakteristik im Jahr 1981

\begin{tabular}{llcc}
\hline & \multicolumn{3}{c}{ Deutsche } \\
& f.s. & t. & durchschn. \\
\hline 1. Faktor & 0,71 & $\mathrm{~h}$ & 5,91 \\
& 0,65 & $\mathrm{~b}$ & 4,98 \\
& 0,64 & $\mathrm{o}$ & 5,56 \\
2. Faktor & 0,63 & $\mathrm{e}$ & 5,81 \\
& 0,75 & $\mathrm{n}$ & 4,78 \\
3. Faktor & 0,66 & $\mathrm{u}$ & 4,24 \\
& 0,56 & $\mathrm{r}$ & 4,52 \\
4. Faktor & 0,83 & $\mathrm{~m}$ & 5,33 \\
& 0,61 & $\mathrm{~s}$ & 5,28 \\
& 0,89 & $\mathrm{p}$ & 5,43 \\
\hline
\end{tabular}

$\mathrm{v} \%=59,8$ 
Tabelle 11

Nationalcharakteristik im Jahr 1991

\begin{tabular}{llcc}
\hline & \multicolumn{3}{c}{ Deutsche } \\
& f.s. & t. & durchschn. \\
\hline 1. Faktor & 0,80 & $\mathrm{~h}$ & 5,41 \\
& 0,68 & $\ddot{\mathrm{o}}$ & 5,51 \\
& 0,65 & $\mathrm{p}$ & 5,04 \\
& 0,47 & $\mathrm{e}$ & 5,16 \\
2. Faktor & 0,45 & $\mathrm{~b}$ & 5,11 \\
& 0,81 & $\mathrm{u}$ & 4,39 \\
& 0,75 & $\mathrm{n}$ & 4,82 \\
3. Faktor & 0,66 & $\mathrm{r}$ & 4,39 \\
& 0,83 & $\mathrm{~s}$ & 5,20 \\
& 0,72 & $\mathrm{~m}$ & 4,98 \\
\hline
\end{tabular}

$\mathrm{v} \%=58,3$

Wobei f.s. $=$ Faktorengewicht, $\mathrm{t}$. $=$ Eigenschaft, durchschn. $=$ Durchschnitt, $\mathrm{v} \%=$ Varianzprozent $\mathrm{b}=$ ehrlich, $\mathrm{e}=$ vernünftig, $\mathrm{h}=$ patriotisch, $\mathrm{m}=$ gebildet, $\mathrm{n}=$ populär, $\ddot{\mathrm{o}}=$ selbstbewusst, $p=$ interessiert für Politik, $r=$ freundlich, $s=$ fleißig, $\mathrm{u}=$ hat Sinn für Humor (HUNYADY 1996, 114) bedeuten.

Ich finde diese Daten für meine Untersuchung wichtig, da auch sie Informationen über die Beurteilung der Deutschen vor und nach den politischen Wenden in Ungarn liefern, wenn auch nicht unter Schülern der allgemeinbildenden Schule, wie in meinen Erhebungen von 1980 und 2005.

Stereotypen können zwar eine beschreibende Wahrheit beinhalten, müssen trotzdem für falsch erklärt werden. Nach Hunyady (HUNYADY 1996, 107) können vier verschiedene Fehler bei den Stereotypen auftreten:

- Ein Durchschnittsmitglied der eigenen Gruppe absolutisiert in seinen Vorstellungen die Erscheinungen im Zusammenhang einer Minderheitsgruppe oder eines ihrer Mitglieder. Der Mensch nimmt naiv, ohne es in Frage zu stellen, an, dass die fremde Gruppe so ist, wie er sie sieht bzw. wie seine eigene Gruppe sie für ihn beschreibt.

- Das Maß des festgestellten Unterschiedes. Die Homogenität der betreffenden Eigenschaft wird innerhalb der eigenen oder der äußeren Gruppe betont, oder das Maß der Überlappungen zwischen den Gruppen wird unterschätzt.

- Das naive Mitglied der eigenen Gruppe nimmt in den Stereotypen über die fremde Gruppe den Kausalnexus falsch wahr.

- Hier geht es um die Relation zwischen dem Inhalt des Stereotypen und dem Hass gegenüber der äußeren Gruppe. Das naive Mitglied der eigenen Gruppe nimmt an, dass sein feindliches Gefühl durch die Eigenschaften der äußeren Gruppe ausgelöst wird. 


\subsection{Ein Vergleich mit anderen Erkenntnissen in Ungarn und in anderen Ländern}

Die europäischen Nachbarn halten die Deutschen vor allem für gut organisiert, akkurat und leicht pedantisch. Das ergab eine Studie der GfK Marktforschung, in der rund 12.000 Bürger in Deutschland, Frankreich, Großbritannien, Italien, den Niederlanden, Österreich, Polen, Russland, Tschechien und der Türkei befragt wurden (SANKTJOHANSER 2006).

Tabelle 12

Was ist deutsch?*

\begin{tabular}{lcc}
\hline Nennungen in \% & Deutschland & $\begin{array}{c}\text { Durchschnitt der Europäer } \\
\text { ohne Deutschland }\end{array}$ \\
\hline $\begin{array}{l}\text { Fleißig und pflichtbewusst } \\
\text { Ordnung, gut organisiert, } \\
\text { pedantisch, genau, akribisch, }\end{array}$ & 23,0 & 4,3 \\
akkurat & 13,1 & 9,8 \\
Pünktlich & 13,1 & \\
Ordnungsliebend, sauber & 12,3 & 2,6 \\
Wirtschaftliche Probleme, & 11,5 & 3,9 \\
Arbeitslosigkeit & & 0,4 \\
Bier & 1,0 & \\
Hitler, Nazis & 0,7 & 5,0 \\
Krieg allgemein & 0,2 & 3,1 \\
\hline
\end{tabular}

*Angaben der Befragten in Prozent auf folgende offene Frage: „Wenn Sie jetzt bitte nun einmal an Deutschland und die Deutschen denken. Was verbinden Sie persönlich mit Deutschland und den Deutschen, wofür stehen ihrer Meinung nach Deutschland und die Deutschen?“ Quelle: GfK-Nürnberg e.V., Mai 2006

Vor der Wiedervereinigung gab es bei den meisten Kindern eine geteilte Auffassung über die „Deutschen“, nämlich die Meinung über den Ostdeutschen und den Westdeutschen. Laut einer soziologischen Studie von Á. Hankiss und K. S. Nagy aus dem Jahre $1980^{2}$ wurden diese „Typen“ von 12jährigen Kindern wie folgt charakterisiert:

Die 'Ostdeutschen' sind Urlauber am Balaton, die mit ihrem Trabi oder Wartburg kommen und auf einem Campingplatz wohnen. Sie wurden von den Kindern stark kritisiert, mit Stereotypen wie: 'Sie lassen uns keinen Platz am Balaton“, „Sie plündern unsere

${ }^{2}$ Forschungsprojekt „Fremdbilder in der Bildschaffung von 12jährigen Kindern. Forschungsinstitut für Massenmedien [Tömegkommunikációs Kutató Központ] 1980. Angaben zitiert in PETNEKI (1994). 
Geschäfte', 'Man kann am Balaton kein ungarisches Wort hören'. Zum Assoziogramm der 'Ostdeutschen' gehören am häufigsten die Begriffe: Balaton, lautes Benehmen, Unhöflichkeit, Wegwerfen von Abfällen, Waldverschmutzung. Dieses 'Bild' über die Bürger der DDR wurde natürlich in den damaligen Lehrbüchern nicht widergespiegelt, man hat stattdessen versucht, mit einer problemfreien und idealisierten Darstellung diesem Bild entgegenzuwirken. Die 'Westdeutschen' und die 'Österreicher' wurden von den Kindern hingegen als friedliche, ruhige und wohlhabende Urlauber gekennzeichnet, die mit guten Autos kommen und in Hotels wohnen. Sie tummeln sich nicht in den Geschäften (Ausnahme: Österreicher in Sopron/Ödenburg), sondern sie schauen sich die Sehenswürdigkeiten an, und sie werden bedient. Die Attribute für die Touristen aus der BRD und Österreich waren gleich: wohlhabend, fährt gutes Auto, höflich und kultiviert in Sinne von 'gut erzogen und gepflegt'. Das Bild der Schweiz kommt in dieser Untersuchung nur im Assoziogramm vor, das Land wird gleichgesetzt mit 'feiner Schokolade'.

(PETNEKi 1994, 120)

Ähnliche Auffassungen über die Angehörigen der verschiedenen deutschsprachigen Länder habe ich ebenfalls 1980 bei meiner Untersuchung unter Absolventen der Grundschule festgestellt. Es zeigte sich deutlich, dass das von den Unterrichtsbehörden offiziell vorgegebene Bild über Deutsche in den Kindern parallel mit den eigenen und aus anderen Informationsquellen gewonnenen Erfahrungen lebte und von ihnen wiedergegeben wurde.

Zehn Jahre nach meiner ersten Untersuchung, also gerade in der Zeit, wo die Grundschulschüler von 1980 ihre weitere Ausbildung beendeten und als junge Arbeitnehmer auf dem Arbeitsmarkt erschienen, hat man unter Erwachsenen eine Untersuchung zu diesem Thema durchgeführt.

Aus einer Erhebung des Ungarischen Instituts für Meinungsforschung (Magyar Közvéleménykutató Intézet) von 1990 geht hervor, dass auf die Frage, mit welchen der aufgezählten Länder Ungarn künftig „,enge Kontakte pflegen sollte, Österreich und die Bundesrepublik mit 93 bzw. 89 von 100 möglichen Sympathienpunkten alle anderen Bewerber um Längen aus dem Rennen“ schlugen (SCHERRER 1991, 271).

\subsection{Aus der Perspektive der Eltern - Deutschsprachige von Erwachsenen eingeschätzt}

Monika Dannerer untersuchte 1992 das in erster Linie durch Geschäftskontakte entstandene Bild bei Erwachsenen. Nach dieser Erhebung sind Österreicher höfliche und angenehme Geschäftspartner, sie orientieren sich aber mehr an Formalismen (Kleidung, Dienstwagen). Sie sind offen, gemütlich, unpünktlich, vergesslich, jovial, schlechte Organisatoren, ausländerfeindlich, nehmen Gesetze, Fristen und Termine nicht so ernst, würden gern die Führerrolle gegenüber den Ungarn spielen.

Deutsche werden für besonders zuverlässig, ruhig, ausgewogen, sehr ordentlich, fleißig, sauber, logisch, korrekt, klar, gesetzestreu, im Ausdruck präzise, ziel- und kundenorientiert, gründlich, höflich, kreativ, gehalten, aber auch für ungeduldig, nicht so offen, kälter, härter und zurückhaltender (Norddeutsche). 
Schweizer werden für ihre Genauigkeit und Zuverlässigkeit geschätzt, aber sie sind überheblich und verstehen die Situation in Ungarn nicht, weil sie immer in Demokratie gelebt und in diesem Jahrhundert keinen Krieg erlebt haben.

Die obigen Ergebnisse ergänzen meine Untersuchungen, da sie bei den Erwachsenen auf persönlichen Erfahrungen und Eindrücken beruhen, und etwa die Meinung der Eltern der im Frühjahr 2005 von mir befragten Schüler der Grundschule vertreten sollen.

\subsection{Auch die Medien tragen zur Herausbildung von Stereotypen bei}

Nach den Angaben der Schüler in meiner Erhebung sind auch die Medien, insbesondere das Fernsehen für sie eine wichtige Informationsquelle über die Deutschen. Es spielt heutzutage eine besonders wichtige Rolle, dass ca. die Hälfte der ungarischen Haushalte verkabelt ist. In Ungarn sind die Sender RTL, RTL2, Sat1, Pro7, 3sat, ORF1 und in Westungarn auch ORF2 zu empfangen. Besonders Kinder sehen viel diese Programme. Den größten Einfluss auf das Bild über Deutsche und Deutschland mögen wohl die folgenden Programme ausüben:

- Krimis (reiche Leute wollen noch reicher werden bzw. ihren Reichtum retten, egal, um welchen Preis) - diese werden oft auch im ungarischen Fernsehprogramm gezeigt.

- Werbung (wichtige Bereiche: Waschen, Putzen, Mode und Schönheitspflege, Getränke, Süßigkeiten usw.). Viele dieser Werbungen werden auch vom ungarischen Fernsehen übernommen (und - wie Katalin Petneki feststellt - leider Gottes wortwörtlich übersetzt).

- Glücksspiele, Quiz (mit wenig Anstrengung kann man große Summen gewinnen) usw.

Diese Sendungen tragen dazu bei, dass ein schematisches Bild über Deutsche entsteht (Unterschiede zwischen Deutschen, Österreichern und Schweizern sind verschwommen) und dass Deutschland für viele ein Traumland wird, wo man leicht reich werden und ohne Sorgen leben kann (PETNEKI 1994, 123).

\section{Die Lehrbücher im Spiegel der gestellten Fragen}

Es konnte bei den gegebenen Antworten kein direkter Zusammenhang zwischen den Kenntnissen bzw. geäußerten Gefühlen der Schüler und ihrer Teilnahme oder NichtTeilnahme an einem Deutschunterricht festgestellt werden. Trotzdem schien es zweckmäßig zu prüfen, wie die in den einzelnen Schulen verwendeten Lehrbücher der deutschen Sprache zu den komplexen Kenntnissen der Schüler über die deutschsprachigen Länder und ihre Bewohner beitragen könnten. Die Sprachbücher wurden in dieser Hinsicht kurz analysiert. 


\subsection{Forderungen an Lehrbücher}

Nach dem Artikel von Katalin Petneki wurde 1979, also im Jahr, als meine erste Untersuchung durchgeführt wurde, dem Deutschunterricht ein dreifaches Ziel gesetzt:

a) ein erzieherisches Ziel,

b) ein sprachlich-kommunikatives Ziel,

c) ein Bildungsziel.

Das so genannte erzieherische Ziel (Herausbildung einer sozialistischen Persönlichkeit) hatte wenig Auswirkung auf die Gestaltung des Unterrichts. Am wichtigsten war das so genannte sprachlich-kommunikative Ziel: Im Mittelpunkt stand die Fremdsprache mit ihrer Lexik und Grammatik. Beim so genannten Bildungsziel stellt es sich heraus, dass man unter Landeskunde eine Summe von Informationen über Geographie, Geschichte, Kultur, Politik, Wirtschaft und Gewohnheiten im Zielsprachenland versteht. Leider wird dabei im Deutschbuch gar nicht beachtet, was die Gymnasiasten in anderen Schulfächern wie Geographie, Geschichte, Literatur, Musik und Kunstgeschichte schon gelernt haben oder lernen werden, so bleiben ihr Weltwissen und ihre Interessen völlig unausgenutzt (PETNEKI 1994, 117-18).

Im erwähnten Artikel wird auch darauf hingewiesen, dass die Landeskunde sowohl bei der Zielsetzung des Unterrichtes als auch bei der Zielsetzung der ungarischen staatlichen Sprachprüfung - und damit der Lernenden selbst - zu kurz komme (PETNEKI 1994, 118).

Petneki bezieht sich auf eine Befragung von Lehrern und Studenten, wonach Landeskunde und literarische Texte im Deutschunterricht bei den Lehrern, wenn auch nicht vorrangig, aber doch höhere Priorität haben, als bei den Studierenden (PETNEKI 1994, 119), welche zwar die Wichtigkeit des Sprachenlernens einsehen, jedoch wenig Wert darauf legen, dass sie auch die mit der Sprache verbundene Kultur kennen lernen. Die Lehrer halten Landeskunde noch für einigermaßen wichtig, sie steht bei ihnen unter den angebotenen elf Aspekten an der 6. Stelle, aber ob sie diese auch im Unterricht entsprechend realisieren, erscheint - laut Petneki - angesichts der Lernerfahrungen der StudentInnen zumindest fraglich. Diese Ansicht hat sich auch bei meiner Untersuchung bestätigt, wo sich herausgestellt hat, dass landeskundliche bzw. die Bildung erweiternde Angebote im Lehrbuch nicht effektiv genug ausgenutzt worden waren.

Einige überregionale Lehrwerke haben versucht, dieses einseitig positive und klischeehafte Bild z.B. mit einer problemorientierten und etwas ironisierenden Darstellung zu bekämpfen (PETNEKI 1994, 120).

\subsection{Ein umfassendes Bild vermitteln - eine Aufgabe auch für Sprachlehrer}

Auch die Sprachlehrer selbst können viel dazu beitragen, dass ihre Schüler sich ein möglichst umfangreiches, reales Bild von den Menschen, welche die zu erlernende Sprache als Muttersprache gebrauchen, schaffen können. Es wurde schon vor über 30 Jahren eine Anforderungsliste an Sprachlehrer in der Schulpraxis erstellt und in der Fachliteratur veröffentlicht: 
1. landeskundlich-semantische und rein landeskundliche Kenntnisse, wie sie die Ziele und Themen des Unterrichts in den entsprechenden Stufen erfordern;

2. die Fähigkeit, sich die unter 1. genannten Kenntnisse zu verschaffen;

3. die Fähigkeit, die unter 1. genannten Kenntnisse auszuwerten und zu interpretieren;

4. die Fähigkeit, die unter 1-3. genannten Kenntnisse und Arbeitsweisen im Rahmen des Fremdsprachenunterrichts zu vermitteln;

5. eine auf Kenntnissen und ihrer Verarbeitung beruhende, objektiv-nüchterne Haltung der Kontakt- und Verständigungsbereitschaft gegenüber dem jeweiligen fremden Volk;

6. die Fähigkeit, die unter 5. genannte Haltung mitzuteilen und zu erklären;

7. die Fähigkeit zu eigener landeskundlicher Forschung auf einem über 2. stehenden Niveau (ERDMENGER \& ISTEL 1978, 84).

Die politische Wende nach 1989 stellte neue Anforderungen und bot zugleich auch den Deutschlehrern neue Möglichkeiten, ihre Aufgaben bezüglich der Vermittlung von Landeskunde zu erfüllen. Die ungarischen Deutschlehrer vor der Wende haben meistens nur die Möglichkeit gehabt, im Rahmen ihres Studiums ein Semester in der Deutschen Demokratischen Republik zu verbringen. Sie konnten zwar während dieses Teilstudiums die deutsche Mentalität in vieler Hinsicht kennen lernen, insbesondere durch Begegnungen mit deutschen Kommilitonen, ihr Aufenthalt wurde aber von offizieller Seite durchpolitisiert. Wie man im westlichen Teil Deutschlands bzw. in Österreich und in der Schweiz lebt, konnten sie in ihrem Studium nicht erleben. Heute kann man schon frei reisen. Die Frage ist nur, ob man ein entsprechendes Stipendium bekommt, oder ob die Eltern einen Studienaufenthalt in einem deutschsprachigen Land finanzieren können. Viele angehende Deutschlehrer sind sich auch dessen nicht genügend bewusst, dass sie in ihrem Unterricht bezüglich der Landeskunde und der Kulturvermittlung eine Doppelrolle erfüllen müssen. Sie sollen in der Lage sein, gegenüber den Schülern die Kultur, Tradition und Gewohnheiten der Deutschen zu vertreten, zugleich aber sollen sie auch die Einstellung und Kenntnisse ihrer Schüler kennen, damit sie gerade die Situationen auflösen können, in denen falsche Stereotypen entstehen oder verstärkt werden können.

Dabei kann viel helfen, wenn die Kinder schon in der Grundschule mindestens einmal im organisierten Rahmen mit deutschen Altersgenossen in Berührung kommen, und wenn die bei diesem Treffen entstandenen eventuellen Konfliktsituationen gemeinsam besprochen und ausgetragen werden können. Es muss den Schülern bewusst gemacht werden, dass die Sprache, die sie erlernen, nicht abstrakt, sondern ein Mittel ist, in einer gegebenen Umgebung zwischenmenschliche Kontakte zu pflegen. Persönliche Begegnungen mit den Vertretern der gelernten Sprache lassen auch Unterschiede in der Metakommunikation bewusst werden. Körpersprachliche Elemente, wie Hände schütteln oder Küsschen geben, können je nach Volk unterschiedlichen Stellenwert haben. Die Unterschiede in den Gewohnheiten können falsche Vorstellungen und Vorurteile hervorrufen. Solange organisierte Begegnung mit Muttersprachlern nicht gewährleistet werden kann, können auch gut ausgewählte Multimedien viel helfen. Gemeinsam angeschaute Filme bieten die Möglichkeit, auch zwischenmenschli- 
che Beziehungen zu analysieren, etwa wie ein alltägliches Familienleben in Deutschland geführt wird oder wie die Beziehungen zwischen Kindern und Eltern sind. Sprachenlernen bedeutet ja auch das Erlernen eines Kommunikationssystems, das von den Muttersprachlern der jeweiligen Sprache benutzt wird.

Die Pädagogik weiß schon seit langem, dass eine stärkere Verbindung von Lernen (mit dem Buch, mit dem Kopf) und praktischem Tun nicht nur mehr Freude macht und stärker motiviert, sondern auch zu besseren Lernergebnissen führt (KRUMM 1991, 4).

Petnekis Auffassung zufolge bestünde eine wesentliche Aufgabe des Fremdsprachenunterrichtes darin, gerade auch die Fremdheit an der Fremdsprache aufzuzeigen. Die Schüler sollten herangeführt werden, damit sie selbst diese Fremdheit wahrnehmen, verstehen und akzeptieren lernen. Dazu sollte die Landeskunde das Handlungsfeld im Fremdsprachenunterricht bieten (PETNEKI 1994, 133).

\subsection{Die Lehrbücher als Informationsquellen}

Hans-Jürgen KRUMM $(1998,100-105)$ zitiert den Stockholmer Kriterienkatalog, wonach - soweit entsprechende Texte und Informationen im Lehrwerk vorhanden sind zu fragen ist, ob diese

- sachlich richtig,

- altersgerecht,

- ausgewogen,

- problemorientiert,

- unterhaltend und

- abwechslungsreich gestaltet sind

- und ob kulturkontrastive Aspekte berücksichtigt werden.

Kriterien bezüglich der Menschen, die im Lehrwerk vorkommen:

- Gibt es Personen, mit denen sich die Schüler identifizieren können?

- Gibt es Personen, für die sich die Schüler interessieren könnten?

- Tauchen männliche und weibliche Personen zu ungefähr gleichen Anteilen im Lehrwerk auf?

- Werden Rollenklischees (z.B. Frau nur als Hausfrau) vermieden? Kriterien im Hinblick auf den Alltag im Lehrwerk:

- Wird das Leben in der Stadt und auf dem Land vorgestellt?

- Wird das Leben in Familie, Schule, bei der Arbeit und in der Freizeit thematisiert?

- Tauchen Menschen aus verschiedenen sozialen Schichten auf?

- Wird das Alltagsleben in allen deutschsprachigen Ländern behandelt? Kriterien zu Geographie und Wirtschaftsleben:

- Enthält das Lehrwerk Karten und Fotos zur Darstellung von Geographie und Wirtschaft (Vielfalt der Landschaftstypen, Industrie etc.)?

Kriterien zur Gesellschaft (politische, wirtschaftliche und gesellschaftliche Verhältnisse): 
- Werden die verschiedenen politischen Systeme der deutschsprachigen Länder vorgestellt?

- Werden die verschiedenen wirtschaftlichen Systeme und Lebensbedingungen in den deutschsprachigen Ländern behandelt?

Kriterien bei der Darstellung des eigenen Landes:

Bietet das Lehrwerk die Möglichkeit,

- die besonderen Beziehungen deutschsprachiger Länder zum eigenen Land zu behandeln?

- über Verhältnisse im eigenen Land, z.B. Sitten und Bräuche, Wirtschaft, soziale Sicherung sich auf Deutsch zu äußern?

Lutz GÖTZE (1994) unterscheidet fünf Lehrwerkkategorien in der zweiten Hälfte des 20. Jahrhunderts. Von diesen fallen die letzten zwei in die Perioden, in denen ich meine Untersuchungen durchgeführt habe.

Für die 3. Generation (70er Jahre), die während meiner Erhebung im Jahre 1980 verwendet wurde, sind nach Götze die folgenden Merkmale charakteristisch:

- ,pragmatische Wende“: soziologischer Ansatz - sprachliches Handeln als Teil sozialen Handelns;

- Sprachhandeln in unterschiedlichen Kommunikationsstrukturen wird geübt; Sprachreflexion anstelle der traditionellen Grammatik;

- häufig wurde Sprachhandeln auf das Einüben von Sprechakten (fragen / antworten; bitten / Bitten erfüllen) reduziert (,Sprechakttheorie“ als linguistische Grundlage in der BRD); in der DDR Versuch, die Sprachakttheorie weiterzuentwickeln: funktionalkommunikative Sprach-beschreibung;

- von allen Funktionen natürlicher Sprache wurde nur noch die kommunikative angesprochen - Sprache verkümmerte zum Informationsträger;

- Überakzentuierung der gesprochenen Sprache.

Die 5. Generation (90er Jahre), die auch bei meiner Erhebung im Jahre 2005 wirkte:

- In Abgrenzung zu Lehrwerken der 3. Generation: Entwicklung aller vier Grundfertigkeiten, vor allem aber auch von Lesen und Schreiben;

- Betonung kognitiver Lehrverfahren (,,mentalistische Wende“);

- Übungen zur Sprachreflexion; interaktive Übungen.

Schauen wir uns nun die einzelnen Lehrbücher an, die die Deutsch lernenden Schüler meiner Erhebung im Unterricht verwenden. Bei den Betrachtungen wollen wir berücksichtigen, dass - wie auch Petneki betont - landeskundliche Inhalte von der Zielgruppe (Alter, Interessen) abhängen sollten, und dass die Aufgabe des Lehrers dabei wäre, die Schüler dazu zu befähigen, die sie interessierenden Inhalte möglichst selbst zu erschließen (PETNEKI 1994, 126).

Hier sollte man sich auch auf eine Umfrage unter Sprachlehrern beziehen, wonach unter 40 Aspekten - der Reihenfolge der Wichtigkeit nach - die Landeskunde den 21., die Interkulturalität den 38. und die Bildung den 40. Platz belegt hat. 


\subsection{Die Sprachbücher}

Im Allgemeinen kann man feststellen, dass alle heute in Ungarn benutzten Sprachbücher für Deutsch als Fremdsprache viel schülerfreundlicher, informativer und alltagsbezogener sind als diejenigen, die bei meiner ersten Erhebung im Jahre 1980 in den Schulen benutzt wurden. Alle Sprachbücher beinhalten Landeskunde, wenn auch in unterschiedlichem Maße bearbeitet, und die meisten bieten auch dem Lehrer die Möglichkeit, berühmte Deutsche kennen lernen zu lassen, auch wenn sie vom Lehrplan her nicht gezwungen wären, dies zu tun.

Geschichte:

4. Klasse: Die Tätigkeit von Martin Luther wird eingehend erörtert. Seine Person und Wirkung gehören zum Grundlehrstoff. Der Name Luther und sein Schaffen sollen also den Schülern der 8. Klasse auch von der Schule her bekannt sein.

5. Klasse: Der vierzigjährigen Herrschaft der österreichischen Kaiserin und ungarischen Königin Maria Theresia - wichtig für die Entstehung der ungarndeutschen Gebiete - wird nicht einmal ein Abschnitt im Lehrbuch gewidmet. Bei der allgemeinen Darstellung der Epoche von Joseph II., der gerade noch kurz behandelt wird, weist der Lehrbuchautor darauf hin, dass entvölkerte ungarische Gebiete mit anderen Volksgruppen, unter anderem auch mit Deutschen besiedelt wurden.

6. Klasse: Die Darstellung des zweiten Weltkrieges mit schockierenden Bildern trägt sicherlich dazu bei, dass sich in vielen Schülern, die wenig andere Eindrücke bekommen, ein einseitig negatives Bild über die Deutschen bildet. Eine Kartenskizze, die Ungarn im zweiten Weltkrieg darstellt, und die die im Bündnis mit Deutschland gewonnenen Gebiete zeigt, weist indirekt darauf hin, dass eine nichtungarische Bevölkerung zum Beispiel um Budapest und im Komitat Baranya lebte.

Das Literaturbuch

Im Lehrbuch für Literatur für die 6. Klasse wird J. W. Goethe behandelt. Thomas Mann aber ist in der 8. Klasse der Entlastung der Schüler zum Opfer gefallen. Im Lehrbuch der Grundschulabsolventen vertritt nur Franz Kafka die deutsche Literatur des 20. Jahrhunderts.

\subsection{Interkulturelle Kompetenz}

Das - nicht nur für den Fremdsprachenunterricht wichtige - Ziel der interkulturellen Kompetenz versteht sich als differenziertes Wissen über die zum Teil ungeschriebenen Verhaltens- und Kommunikationsmuster verschiedener Gesellschaften. Schullehrpläne sollten sich dementsprechend an einer Sensibilisierung gegenüber anderen Kulturen orientieren, um Schüler auf einen Austausch der Kulturen vorzubereiten. In diesem Band findet man Beiträge aus Schule sowie Wirtschaft, die Fallbeispiele präsentieren und zur Diskussion anregen (VOLKMANN et al. 2002).

Grundsätzlich spielen auch für interkulturelles Lernen alle Methoden kommunikativen Fremdsprachenunterrichts eine Rolle, da dieses in den Fremdsprachenunterricht 
integriert ist. Einige spezielle methodologische Zugangsweisen, die zum Erreichen interkultureller Lernziele dienen können, hat Krumm wie folgt zusammengefasst:

a) gezieltes Wahrnehmungstraining und Sensibilisierung für eigenkulturelle Prägungen (Lehrwerke „Sichtwechsel“, „Sprachbrücke“);

b) bewusste Konfrontation / bewusster Vergleich eigenkultureller Prägungen und Manifestationen mit den kulturellen Manifestationen der deutschsprachigen Länder;

c) Bedeutungsrecherchen und Bedeutungscollagen;

d) kulturkontrastive Erfahrungen sammeln: eigene Erfahrungsberichte;

e) Vorwissen aktivieren: Assoziogramme; Recherchen (KRUMM 1992, 19).

Um die auch oben genannten Ziele zu erreichen, schlägt Professor Hans-Jürgen Krumm Projektarbeiten in der Schule vor. Nach Twellmann ist unter Projekt im Rahmen schulischen Unterrichts ein Vorhaben zu verstehen, das von Lehrern und Schülern gemeinsam getragen und verantwortet wird und das sich durch eine begrenzte Bezogenheit auf die Gesellschaft auszeichnet. Dieser Gesellschaftsbezug wird vor allem deutlich im Ergebnis des gemeinsamen Vorgehens, das irgendwie gesellschaftlich relevant, also „einsetzbar“ und „benutzbar“" sein soll.

Krumm stellt das Projekt als festen, integrierten Bestandteil des Sprachunterrichts vor - nicht als Alternative zu allem, was sonst im Unterricht passiert, sondern als Unterrichtsprinzip, das sich mit den vorhandenen Lehrplänen und Lehrbüchern verbinden lässt.

Unterrichtsprojekte sind durch die folgenden Merkmale gekennzeichnet:

1. ein konkretes Ziel, das es erlaubt, Sprache in kommunikativer Funktion zu verwenden und das es erlaubt, Neues, Fremdes zu entdecken und zu erfahren;

2. gemeinsame Planung und Ausführung durch Lehrer und Schüler, wobei zunächst einmal die Schüler versuchen, mit ihren vorhandenen Sprachkenntnissen zurechtzukommen; der Lehrer ist der sprachliche und sachliche Helfer; der Sprachunterricht liefert diejenigen sprachlichen Mittel, die zur Bewältigung der Aufgabe gebraucht werden;

3. die Hereinnahme der Außenwelt in den Unterricht bzw. die Erweiterung des Unterrichts in die Außenwelt hinein, wobei die Einheit von Sprache und Handeln, von Sprache und Situation konkret erfahrbar wird;

4. die selbständige Recherche und Aktion der Schüler unter Benutzung aller verfügbaren Hilfsmittel, zu denen z.B. Wörterbücher und Grammatiken ebenso gehören wie evtl. Schreibmaschine, Computer, Mikrophon oder Kamera;

5. ein präsentables Ergebnis, das auch über das Klassenzimmer hinaus als Poster, Zeitung, Korrespondenz, Aufführung oder Ähnliches vorgezeigt werden kann und im günstigsten Fall (z.B. bei der Klassenkorrespondenz) weitere Aktionen nach sich zieht; Spracharbeit (d.h. Fehlerkorrektur, Grammatikarbeit, Schreiben) vollzieht sich dabei in Form der Überarbeitung und Verbesserung des Produktes, d.h. die Klasse wird, wie von Freinet gefordert, zur „Werkstatt“ (KRUMM 1991, 6).

Ähnliche Projektarbeiten lassen sich auch in Ungarn durchführen. Insbesondere scheinen dafür Vorbereitungen zum Empfang einer Partnerklasse aus Deutschland, 
oder zum Besuch in einem deutschsprachigen Land geeignet zu sein. Professor Krumm fasst in fünf Punkten zusammen, warum Projektarbeiten so wichtig sein können:

1. Der Sprachunterricht ist für die Schüler in der Regel die einzige kommunikative Situation, in der sie erfahren können, wofür man die Fremdsprache gebrauchen kann. Wenn wir auf diesen Anwendungsbezug verzichten, bleibt der Lernzweck für die Schüler unklar und der Grund für das Lernen wird in weite Ferne gerückt - und damit schwindet auch die Motivation. Der Sprachunterricht muss für die Schüler zum ,kommunikativen Ernstfall“" werden.

2. Sprachunterricht hängt enger als andere Unterrichtsfächer mit der Entwicklung von Selbstbewusstsein zusammen: Die Schüler werden im Deutschunterricht oft so behandelt, als wüssten sie nicht, wie man einkauft, zur Post geht usw. Dabei wissen und können sie das alles schon, sie müssen nur noch lernen, dies auch im Medium der Fremdsprache zu tun. Ein ausschließlich auf das Lehrbuch fixierter Unterricht aber erlaubt den Schülern nicht, ihre eigenen Erfahrungen und Kenntnisse einzubringen.

3. Unter dem Gesichtspunkt der Lernmotivation ist die Neugier, die Möglichkeit, im Rahmen des Erlernens einer Fremdsprache Fremdes zu erkunden, ein wichtiger Faktor: Landeskunde wird so zu einem interessanten Erfahrungsfeld, das nicht über trockenes angelerntes Wissen, sondern über eigene Erfahrungen erschlossen wird. Ohne Realitätsbezug lässt sich eine Sprache gar nicht angemessen lernen, denn erst im außerunterrichtlichen Kontext gewinnen die Wörter ihre kontextspezifische Bedeutung.

4. Lernpsychologische Argumente: Begreifen durch Handeln, die Einbeziehung ganzheitlicher Lernerfahrungen aktivieren, führt zu wirkungsvollerem Lernen als das reine Buchlernen.

5. Das Erlernen von Mitbestimmung ist nicht nur im Hinblick auf die allgemeinen Erziehungsziele, sondern ganz konkret für den Sprachlernerfolg selbst wichtig: Nur wer begreift, dass er für sein Sprachenlernen selbst mitverantwortlich ist, wird eine Sprache erfolgreich lernen, eigene Sprachstrategien entwickeln und in der Lage sein, über die Unterrichtszeit hinaus die Sprache weiter zu lernen. (KRUMM 1991, 6)

Eine kürzlich erschienene Studie des von dem ungarischen Staatsoberhaupt einberufenen Weisenrates stellt fest: Im Inhalt des schulischen Unterrichtes ist nicht nur die Proportion zwischen dem Mentalen und Somatischen deformiert, sondern charakteristisch ist auch die Dominanz der linken Gehirnhälfte innerhalb der mentalen Förderung.

In dieser befinden sich die Zentren für:

- Sprechen und Verstehen ( Kommunikation - Textverstehen)

- logisches, rationales, analytisches Denken

- Zeit und Rhythmengefühl. In der rechten Gehirnhälfte sitzen:

- die emotionellen Akzente des Sprechens (soziale Beziehungen, Verhaltensnormen)

- das Erkennen des Gesehenen, Raumsicht (visuelle Kultur, Phantasie)

- die Wahrnehmung von Musik (die Vielfältigkeit der echten Gefühle) 
- das synthetische Denken

- die Kreativität (nur die rechte Hemisphäre hat Humor)

Eine harmonische Zusammenarbeit zwischen den beiden Hemisphären ist nur möglich, wenn beide Hälften ausgeglichen entwickelt werden. Dies bedeutet zugleich auch die Förderung der seelischen Gesundheit.

Die obige Forderung, wonach in der schulischen Erziehung auch die rechte Gehirnhälfte, die unter anderem auch für das synthetische Denken und die Kreativität zuständig ist, entsprechend gefördert werden soll (ÓNÓDI 2009, 114), wird auch von den oben dargestellten Untersuchungen unterstrichen, da viele der befragten Schüler Probleme hatten, bei der Beantwortung einzelner Fragen ihre Kenntnisse aus verschiedenen Lehrfächern zu synthetisieren.

\section{Referenzen}

ERDMENGER, M. \& H.W. Istel (1978) Didaktik der Landeskunde (München: Hueber).

GöTZE, L. (1994) 'Fünf Lehrwerkgenerationen' in B. KAST \& G. NEUNER, Hrsg., Zur Analyse, Begutachtung und Entwicklung von Lehrwerken (Berlin \& München: Langenscheidt) 29-30.

HuNYADY, GY. (1996) Sztereotípiák a változó közgondolkodásban (Budapest: Akadémiai).

KÖNIG, R., Hrsg. (1971) Das Fischer-Lexikon - Soziologie (Frankfurt am Main: Fischer-Taschenbuch)

KRUMM, H-J. (1991) Unterrichtsprojekte - praktisches Lernen im Deutschunterricht, April, 1991: Fremdsprache Deutsch (München: Klett).

KRUMM, H-J. (1992) 'Bilder im Kopf: Interkulturelles Lernen und Landeskunde', Fremdsprache Deutsch 6, 16-20.

KRUMM, H-J. (1994) 'Stockholmer Kriterienkatalog' in B. KAST, Hrsg., Zur Analyse, Begutachtung und Entwicklung von Lehrwerken für den fremdsprachlichen Deutschunterricht (Berlin \& München: Langenscheidt) 100-105.

ÓNÓDI, Sz. (2009) 'Kompetenciák, kiemelt területek: Szárny és teher - A magyar oktatás helyzetének elemzése' heruntergeladen am 15. Februar 2010 von http://bolcsektanacsa. solyomlaszlo.hu/index.html.

Petneki, K. (1994) 'Gedanken über die Rolle der Landeskunde im ungarischen Deutschunterricht' in A. MÁdL \& CH. SCHWIEDERSKI, Hrsg., Jahrbuch der ungarischen Germanistik (Bonn: DAAD \& Budapest: GuG) 117-35.

SANKTJOHANSER, A. (2006) 'Was ist typisch deutsch?' heruntergeladen am 9. November 2007 von http://www.focus.de/wissen/bildung/deutsch/stereotype_aid_21930.html.

SCHERRER, S. (1991) 'Die Attraktivität der Langeweile - Ungarn buhlen um die Gunst der Deutschen' in G. Trautmann, Hrsg., Die hässlichen Deutschen? Deutschland im Spiegel der westlichen und östlichen Nachbarn (Darmstadt: Ausblicke).

VolKMAnN, L., K. STIERSTORFER \& W. GehrInG, Hrsg. (2002) Interkulturelle Kompetenz: Konzepte und Praxis des Unterrichts (Tübingen: Narr). 